\title{
Trailing-edge noise generation from a flat-plate aerofoil interacting with a prescribed vortex
}

\author{
Jacob M. Turner \& Jae Wook Kim \\ Institute of Sound and Vibration, University of Southampton \\ Southampton, SO17 1BJ, United Kingdom
}

\begin{abstract}
Aerodynamic noise generated by aerofoil-vortex interaction has widely been studied in the past where the primary noise source mechanism is the scattering of the vortex at the leading edge (LE) of the aerofoil. In this paper the secondary source mechanism - the subsequent vortical scattering at the trailing edge (TE) - is investigated in detail, which shows that the secondary source mechanism is mainly contributed by nonlinear effects as predicted by some analytical studies in the past. The present study is performed by employing high-resolution numerical simulations based on a prescribed non-singular vortex impinging on a flat-plate aerofoil with zero mean loading. The present work investigates both inviscid and viscous flow conditions. The inviscid flow condition is intended to support and extend from the existing theoretical works, whereas the viscous one leads to more realistic findings. The current viscous study involves laminar boundary layers and their convective instability travelling with the impinged vortex. One of the most notable observations made in this work is that the vortical scattering at the TE (in the absence of turbulent boundary layers) may become a dictating source of noise at high frequencies (surpassing the primary source at the LE) across a wide range of observer angles for both inviscid and viscous flow conditions. It is found that the high-frequency dominant source is produced by secondary near-wall vortices that are induced as a result of nonlinear interactions between the aerofoil and the impinging vortex. This new discovery makes a contrast to the existing knowledge on aerofoil-vortex interaction noise in which the secondary source is normally assumed inferior to the primary at all frequencies.
\end{abstract}

Keywords: Aerofoil-vortex interaction noise; Trailing-edge noise; Computational aeroacoustics

\section{Introduction}

An aerofoil interacting with upstream vortical disturbances is considered one of the fundamental sources of aerodynamic noise and has been investigated extensively through a wide range of approaches. This includes the pioneering theoretical work of Amiet [1] for a flat-plate aerofoil subjected to a frozen turbulent stream [2 4], further theoretical work based on harmonic gusts [5] 9], wind-tunnel experiments [10-12] and various numerical simulations [13 21. The majority of the aerofoil noise due to upstream vortical disturbances emanate from the leading edge (LE) where the impinging vortices/eddies scatter into acoustic waves, which is the primary source mechanism. The secondary source mechanisms are associated with the existence of a trailing edge (TE) and are twofold: $(a)$ the backscattering of the acoustic waves produced earlier (travelling back and forth between the LE and TE); and, (b) the scattering of the vortices at the TE (after being bisected 
and modified at the LE). Herein, the primary source mechanism is denoted by LEVS (LE vortical scattering) and the two secondary source mechanisms by ABS (acoustic backscattering) and TEVS (TE vortical scattering) in this paper. For those who are familiar with aerofoil "self-noise" (due to turbulent boundary layer scattered at the TE), it should be noted here that we are focusing on "interaction noise" in this paper and therefore the self-noise contribution is purposely excluded - no turbulent boundary layer is considered.

A comprehensive level of understanding has been made on the ABS event through the work of Rienstra [22], Ayton and Peake [23], Roger et al. [24]. In comparison, there is a gap in knowledge with regard to the TEVS event, and it is therefore the main focus of this paper. One of the earliest theoretical works to consider this problem was carried out by Crighton [25], who considered the sound generated by a point vortex turning around the $\mathrm{TE}$ of a semi-infinite plate in a low-speed flow. In addition, Howe [26, 27] also conducted a theoretical work on this subject based on a point vortex travelling over a flat-plate aerofoil at a certain vertical offset, where he demonstrated the importance of the unsteady Kutta condition at the TE. With the unsteady Kutta condition implemented, he showed that an additional distribution of shed vorticity was produced in the wake. As long as the wake velocity was the same as the mean flow speed, Howe showed that the shed vortex sheet effectively eliminated the acoustic source (scattering of the original vortex) at the TE. This theoretical result was later reiterated by Glegg and Devenport [28] for aerofoils of arbitrary thickness utilising a generalised form of the Blasius theorem. These theoretical results are based on linear perturbations, which are usually considered reasonable if the vortex is sufficiently weak or its passage sufficiently distant from the aerofoil. A rule of thumb suggested by Grace [29] is that the vortex induced velocity should remain less than $n \%$ of the mean flow speed for a vertical offset of $n \%$ of the aerofoil semi-chord in order for the linearity assumption to be applicable.

When nonlinear effects are introduced, additional sound is generated at the TE as Howe [26] anticipated. This has been shown mathematically by Abou-Hussein et al. [30] who extended the classical work of Howe [26] to account for the image vortex contribution on the trajectory of the bypassing point vortex. Also, an enhanced model of the shed vortex sheet based on the Brown and Michael equation 31] was developed by Howe [32. This model was applied to a Joukowski aerofoil [33] and a flat plate undergoing a heaving motion [34. These relatively recent theoretical studies based on a point vortex have neglected some of the physical events that may have a significant impact on the sound generation mechanisms. The point vortex (singularity) means that only one-way interaction is available from the vortex to the aerofoil but not the reverse (apart from the trajectory of the vortex). In reality, a distributed (non-singular) vortex can create diverse interactions with the aerofoil such as a direct collision between them resulting in a completely different vorticity distribution (including small-scale near-wall vortices produced as a result of the collision) travelling over the aerofoil. In such a scenario, a significant level of vortical scattering into sound is expected to appear at the TE.

In rotorcraft research community, there exist some experimental measurements showing the signature of TEVS that appeared during blade-vortex interactions (BVI). Straus et al. [35] directly compared their measured lift with the linear theory of Sears [36] and observed a major discrepancy between them approximately when the vortex passed the TE. Horner et al. 37] reported a noticeable fluctuation in the time history of aerodynamic forces and moment when the vortex crossed the TE. Kitaplioglu et al. [38] also made the same observation using surface pressure transducers and in this case they even captured an upstream travelling pressure wave that was synchronised with the vortex crossing at the TE. Similar results were also obtained from numerical simulations carried out by 
Bridgeman et al. [39] and Thom and Duraisamy [40. The strength of the vortices used in these rotorcraft BVI studies was quite substantial. For example, the vortex-induced velocity perturbation was as high as 30\% of the freestream velocity in Straus et al. [35] and even $55 \%$ in Thom and Duraisamy [40. This implies that there was a sufficient level of nonlinear effects involved in the rotorcraft BVI studies which might have resulted in the TEVS noise signatures. However, detailed information (such as frequency contents) of the TEVS event has not been reported in the previous studies that are limited to the time domain focusing mainly on the aerodynamic forces.

This paper aims to provide an enhanced understanding of the secondary noise sources, with particular interest in TEVS, appearing in the event of aerofoil-vortex interaction. For this purpose high-resolution numerical simulations are performed, from which critical high-frequency contents of the secondary noise sources are accurately captured. We choose a zero-thickness flat-plate aerofoil at zero incidence angle to the base flow (prior to interacting with a vortex) for this fundamental study. We use a divergence-free distributed vortex with a finite core (as opposed to a point vortex) superimposed onto the base flow (upstream of the aerofoil) as an initial condition. The vortex is constructed from a Gaussian shape function which provides a broadband frequency spectrum. Both inviscid and viscous (laminar boundary layer) solutions are provided in this paper to understand the relative difference, which has not been attempted in the past. Also, parametric studies are conducted with regard to the initial vortex strength and the vertical offset between the vortex and the aerofoil. One of the most remarkable findings in this study is that the TEVS noise often becomes the most dominant source at high frequencies over a wide range of observer angles (surpassing the primary LEVS noise) which is contradictory to the existing knowledge. This new outcome turns out to be a consequence of nonlinear source effects due to induced near-wall vortices.

The paper is organised in the following order. Section 2 provides an overview of the current problem and computational set-up including some details of the numerical methods and the prescribed vortical disturbance used in this work. In Section 3 some significant characteristics of the secondary noise sources, including the high-frequency dominance of the TEVS noise, found in the inviscid flow condition are introduced and discussed. Section 4 provides in-depth investigations into the high-frequency TEVS noise in relation with the induced near-wall vortices. In Section 5 the effect of viscosity is investigated in order to understand how it changes/maintains the high-frequency TEVS noise in the presence of laminar boundary layers. Finally some concluding remarks are made in Section 6 .

\section{Description of problem and the computational set-up}

A schematic illustration of the current aerofoil-vortex interaction problem is shown in Fig. 1. In this canonical study, we employ a flat plate as a representation of a thin aerofoil which has historically been adopted in many theoretical, experimental and computational studies to date. Instantaneous snapshots of the sound waves generated as a result of the interaction are shown in Fig. 2, obtained from the current numerical simulations. The figure compares two different cases: one with a semi-infinite-chord aerofoil and the other with a finite chord. The semi-infinite-chord case (Fig. 2a) displays the LEVS (leading-edge vortical scattering) noise that is the primary component of the aerofoil-vortex interaction noise. On the other hand, the finite-chord case (Fig. $2 b$ ) reveals the secondary components of the noise (as well as the primary) generated due to the presence of the trailing edge, i.e. TEVS (trailing-edge vortical scattering) and ABS (acoustic backscattering), as indicated earlier. The TEVS noise is the main subject of this paper. 
(a)

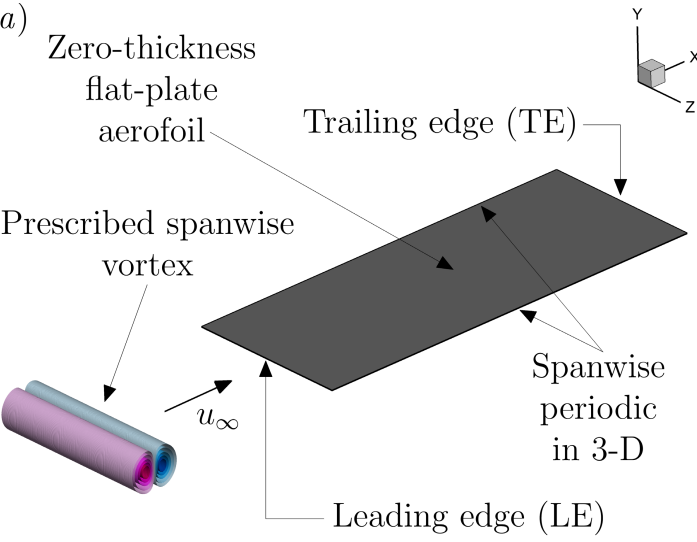

(b)

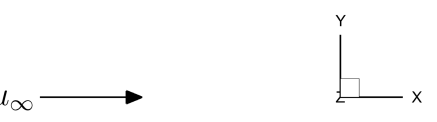

$y_{0}$ (vertical offset)

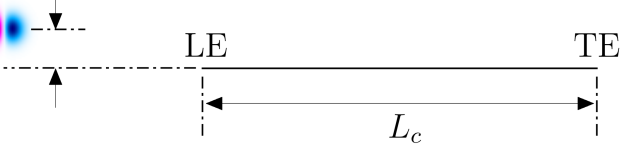

(Side view)

Figure 1: Schematic diagram of the current aerofoil-vortex interaction problem: $(a)$ an isometric view and (b) a side view.
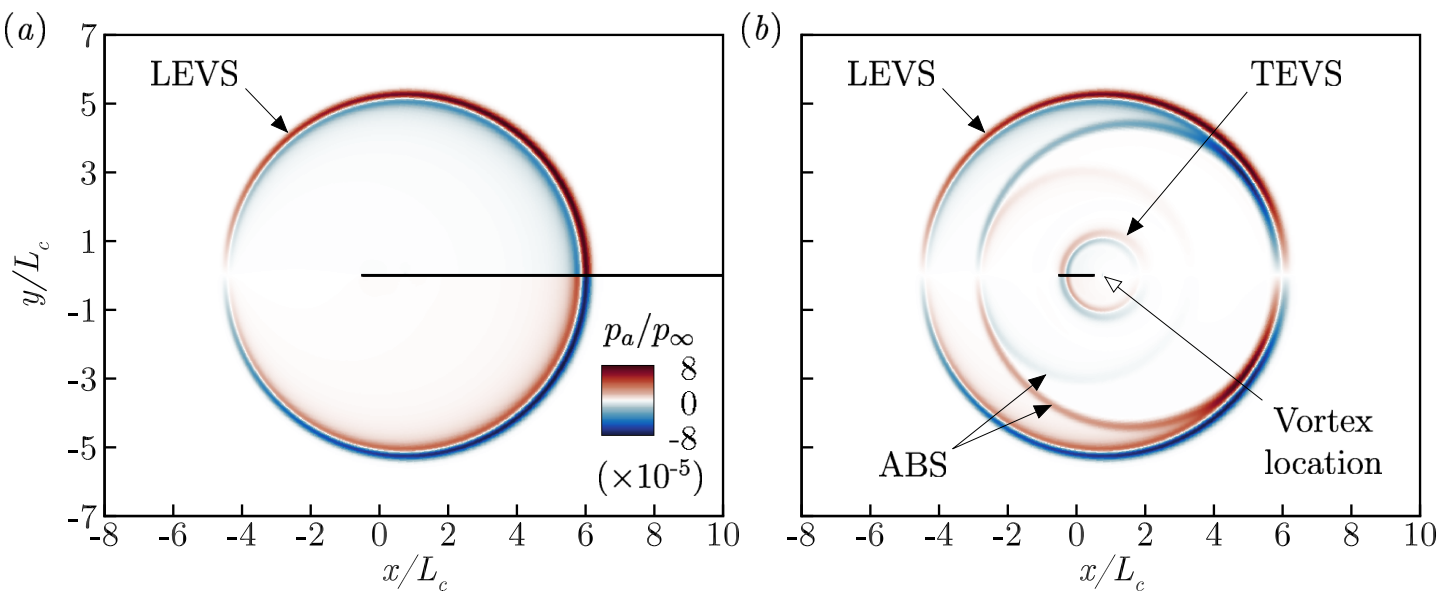

Figure 2: Instantaneous contour plots of acoustic pressure $\left(p_{a} / p_{\infty}\right)$ obtained from the current simulations at the time of the impinged vortex reaching $x / L_{c}=0.7$, for two different cases: $(a)$ a semi-infinite and $(b)$ a finite aerofoil; an inviscid base flow of $M_{\infty}=0.24$ and zero incidence angle is used where the impinging vortex has zero vertical offset from the aerofoil surface; the acronyms are defined as follows - LEVS: leadingedge vortical scattering; TEVS: trailing-edge vortical scattering; and, ABS: acoustic backscattering.

\begin{tabular}{cccccc}
\hline Case & $R e_{\infty}$ & $2-\mathrm{D} / 3-\mathrm{D}$ & $\epsilon$ & $y_{0} / L_{c}$ & $\max |v|_{t=0} / u_{\infty}$ \\
\hline 1 & inviscid & $2-\mathrm{D}$ & $\epsilon_{0}$ & 0 & 0.025 \\
2 & inviscid & $2-\mathrm{D}$ & $\epsilon_{0} / 2$ & 0 & 0.0125 \\
3 & inviscid & $2-\mathrm{D}$ & $\epsilon_{0} / 4$ & 0 & 0.00625 \\
4 & inviscid & $2-\mathrm{D}$ & $\epsilon_{0}$ & 0.03 & 0.025 \\
5 & inviscid & $2-\mathrm{D}$ & $\epsilon_{0}$ & 0.06 & 0.025 \\
6 & $4 \times 10^{5}$ & 3 -D & 0 & (base flow) & 0 \\
7 & $4 \times 10^{5}$ & 3 -D & $2 \epsilon_{0}$ & 0 & 0.05 \\
8 & $4 \times 10^{5}$ & 3 -D & $4 \epsilon_{0}$ & 0 & 0.1 \\
9 & $4 \times 10^{5}$ & $3-\mathrm{D}$ & $6 \epsilon_{0}$ & 0 & 0.15 \\
\hline
\end{tabular}

Table 1: The current simulation cases for various flow conditions; the Reynolds number is defined as $R e_{\infty}=\rho_{\infty} u_{\infty} L_{c} / \mu_{\infty}$; the parameters $\epsilon$ and $y_{0}$ represent the vortex strength and vertical offset, respectively - see Eq. (6) and Fig. 1 $(b) ; \max |v|_{t=0}$ means the largest velocity perturbation (amplitude) induced by the impinging vortex at the initial condition; $L_{c}$ is the chord of the finite aerofoil, set as the reference unit length of the current simulations. 
A total of 9 different flow conditions are explored in the current work, comprising two-dimensional (2-D) inviscid and three-dimensional (3-D) viscous cases. The list of the current simulation cases is provided in Table 1. Cases 1 to 5 present the ideal inviscid solutions (as shown in Fig. 2p that can be discussed and compared against the existing analytical solutions. The list contains parametric tests for investigating the effects of different strengths and vertical offsets of the impinging vortex. Cases 6 to 9 present more realistic 3-D viscous solutions based on laminar boundary layers. It is shown later that the effect of viscosity on the aerofoil-vortex interaction and the subsequent TEVS noise is significant. It is worth noting that the vertical offsets are not included in the viscous cases based on the findings in Section 4.3. In each case, two separate simulations - one with a finite aerofoil and the other with a semi-infinite aerofoil - are carried out in order to split the primary and secondary sources. In addition, auxiliary simulations with an inverse semi-infinite aerofoil are performed in the inviscid cases in order to extract pure TEVS and ABS sources (to follow in Section 3). Therefore, a total of 23 individual (15 inviscid and 8 viscous) simulations are carried out for this study.

\subsection{Computational domain and aerofoil geometry}

The computational domain is a rectangular plane (or a cuboid in the 3 -D viscous cases) containing a flat-plate aerofoil at the centre with zero thickness and zero incidence angle (against the base flow). The zero-thickness aerofoil is modelled by using a H-topology grid system where the central branch cut represents the aerofoil's upper and lower surfaces with no gap between them. The longitudinal and vertical boundaries of the domain are surrounded by a sponge layer through which the flow is (gently) forced to maintain the required mean flow condition. Any acoustic waves are attenuated and absorbed in the sponge layer to prevent numerical reflections at the outer boundaries. The entire computational domain; the inner region (physical domain) where meaningful simulation data are obtained; and, the sponge layer zone are defined as

$$
\left.\begin{array}{c}
\mathcal{D}_{\infty}=\left\{\boldsymbol{x} \mid x / L_{c} \in[-7,11], y / L_{c} \in[-7,7]\right\}, \\
\mathcal{D}_{\text {physical }}=\left\{\boldsymbol{x} \mid x / L_{c} \in[-5,5], y / L_{c} \in[-5,5]\right\}, \\
\mathcal{D}_{\text {sponge }}=\mathcal{D}_{\infty}-\mathcal{D}_{\text {physical }},
\end{array}\right\}
$$

where $L_{c}$ denotes the chord length of the finite-chord aerofoil. The aerofoil's leading and trailing edges are located at $x / L_{c}=-1 / 2$ and $1 / 2$, respectively. In the 3 -D viscous cases, a spanwise domain size of $z / L_{c} \in[-1 / 40,1 / 40]$ (i.e. $5 \%$ of the chord) is used and spanwise periodic boundary conditions are implemented.

\subsection{Governing equations and numerical methods}

The current work employs 2-D and 3-D compressible Euler and Navier-Stokes equations in order to cover the various cases listed in Table 1. The full 3-D Navier-Stokes equations (with a source term for the sponge layer mentioned earlier) can be written in a conservative form, transformed onto a generalised coordinate system as

$$
\frac{\partial}{\partial t}\left(\frac{\boldsymbol{Q}}{J}\right)+\frac{\partial}{\partial \xi_{i}}\left(\frac{\boldsymbol{E}_{j}-R e_{\infty}^{-1} M_{\infty} \boldsymbol{F}_{j}}{J} \frac{\partial \xi_{i}}{\partial x_{j}}\right)=-\frac{a_{\infty}}{L_{c}} \frac{\boldsymbol{S}}{J},
$$

where the indices $i=1,2,3$ and $j=1,2,3$ denote the three dimensions; and, $a_{\infty}$ is the ambient speed of sound. The vectors of the conservative variables, inviscid and viscous 
fluxes are given by

$$
\left.\begin{array}{c}
\boldsymbol{Q}=\left[\rho, \rho u, \rho v, \rho w, \rho e_{\mathrm{t}}\right]^{T}, \\
\boldsymbol{E}_{j}=\left[\rho u_{j},\left(\rho u u_{j}+\delta_{1 j} p\right),\left(\rho v u_{j}+\delta_{2 j} p\right),\left(\rho w u_{j}+\delta_{3 j} p\right),\left(\rho e_{\mathrm{t}}+p\right) u_{j}\right]^{T}, \\
\boldsymbol{F}_{j}=\left[0, \tau_{1 j}, \tau_{2 j}, \tau_{3 j}, u_{i} \tau_{j i}+q_{j}\right]^{T},
\end{array}\right\}
$$

with the stress tensor and heat flux vector written as

$$
\tau_{i j}=\mu\left(\frac{\partial u_{i}}{\partial x_{j}}+\frac{\partial u_{j}}{\partial x_{i}}-\frac{2}{3} \delta_{i j} \frac{\partial u_{i}}{\partial x_{i}}\right), \quad q_{j}=\frac{\mu}{(\gamma-1) \operatorname{Pr}} \frac{\partial T}{\partial x_{j}},
$$

where $\xi_{i}=\{\xi, \eta, \zeta\}$ are the generalised coordinates, $x_{j}=\{x, y, z\}$ are the Cartesian coordinates, $\delta_{i j}$ is the Kronecker delta, $u_{j}=\{u, v, w\}, e_{\mathrm{t}}=p /[(\gamma-1) \rho]+u_{j} u_{j} / 2$ and $\gamma=1.4$ for air. The local dynamic viscosity $\mu$ is calculated by using Sutherland's law [41]. In the current set-up, $\xi, \eta$ and $\zeta$ are aligned in the streamwise, vertical and lateral directions, respectively. The Jacobian determinant of the coordinate transformation (from Cartesian to the generalised) is given by $J^{-1}=|\partial(x, y, z) / \partial(\xi, \eta, \zeta)|$ [42]. The extra source term $\boldsymbol{S}$ on the right-hand side of Eq. (2) is non-zero within the sponge layer only, which is described in Kim et al. [43, 44. In this paper, the free-stream Mach and Reynolds numbers are defined as $M_{\infty}=u_{\infty} / a_{\infty}$ and $R e_{\infty}=\rho_{\infty} u_{\infty} L_{c} / \mu_{\infty}$. The Mach number is fixed at $M_{\infty}=0.24$ and a Reynolds number of $R e_{\infty}=4 \times 10^{5}$ is considered as listed in Table 1. The governing equations are non-dimensionalised based on the aerofoil chord length $L_{c}$ for length scales, the ambient speed of sound $a_{\infty}$ for velocities, $L_{c} / a_{\infty}$ for time scales and $\rho_{\infty} a_{\infty}^{2}$ for pressure. Temperature, density and dynamic viscosity are normalised by their respective ambient values: $T_{\infty}, \rho_{\infty}$ and $\mu_{\infty}$.

The governing equations given above are solved by using high-order accurate numerical methods specifically developed for aeroacoustic simulations on structured grids. The flux derivatives in space are calculated based on fourth-order pentadiagonal compact finite difference schemes with seven-point stencils [45]. Explicit time advancing of the numerical solution is carried out by using the classical fourth-order Runge-Kutta scheme with the CFL number of 0.95. Numerical stability is maintained by implementing sixth-order pentadiagonal compact filters for which the cutoff wavenumber (normalised by the grid spacing) is set to $0.85 \pi$ [46]. In addition to the sponge layers used, characteristics-based non-reflecting boundary conditions [47] are applied at the far-boundaries in order to prevent any outgoing waves from returning to the computational domain. Periodic conditions are used across the spanwise boundary planes as indicated earlier (for the 3 -D cases). On the aerofoil surface, slip (no penetration) and no-slip wall boundary conditions are implemented for the inviscid and viscous cases, respectively [48]. Additionally, the Kutta condition is maintained at the trailing edge.

The current numerical simulations are performed on a structured grid that is moderately stretched from the aerofoil to the far field (uniform in span in the 3-D cases), with the smallest cells uniformly positioned on the aerofoil surface. A sufficiently high level of grid resolution is maintained throughout the domain (except in the sponge zone) in order to directly capture the broadband sound spectra radiated in the far field, without an interference from numerical background noise. The number of grid cells used are $\left(N_{x}, N_{y}, N_{z}\right)=(1600,960,-)$ in the inviscid cases and $(2240,1340,38)$ in the viscous cases. The smallest cell sizes used on the aerofoil surface are $\Delta(x, y, z)_{\min } / L_{c}=(2,2,-) \times 10^{-3}$ in the inviscid cases and $(1.43,0.28,1.32) \times 10^{-3}$ in the viscous cases. The validation of the current grid resolution is provided in Appendix A

The computation is parallelised via domain decomposition and message passing interface (MPI) approaches. The compact finite difference schemes and filters used are implicit 
in space due to the inversion of pentadiagonal matrices involved, which requires a precise and efficient technique for the parallelisation in order to avoid numerical artefacts that may appear at the subdomain boundaries. A recent parallelisation approach based on quasi-disjoint matrix systems [49] offering super-linear scalability is used in the present paper. The entire domain is decomposed and distributed onto 1024 or 512 separate processor cores. The parallel computation has successfully been carried out in the UK national supercomputer ARCHER as well as in IRIDIS5 cluster at the University of Southampton.

\subsection{Prescribed spanwise vortex model}

The current study employs a spanwise-uniform vortex model prescribed as an initial condition. The vortex model is based on a Gaussian shape function suggested by Yee et al. [50], which provides a continuous divergence-free velocity field (as opposed to a theoretical point vortex):

$$
\{u(\boldsymbol{x}), v(\boldsymbol{x})\}=a_{\infty} \psi(\boldsymbol{x})\left\{M_{\infty}+\sigma \frac{y-y_{0}}{L_{c}},-\sigma \frac{x-x_{0}}{L_{c}}\right\},
$$

where $\left(x_{0}, y_{0}\right)$ is the initial location of the vortex and in particular $y_{0}$ represents the vertical offset of the vortex from the aerofoil surface. In this paper, $x_{0}=-L_{c}$ is fixed and $y_{0}=0$ (zero offset) is the default case unless otherwise stated. The Gaussian shape function is defined as

$$
\psi(\boldsymbol{x})=\frac{\epsilon}{2 \pi} \exp \left[\frac{1}{2}-\sigma^{2} \frac{\left(x-x_{0}\right)^{2}+\left(y-y_{0}\right)^{2}}{2 L_{c}^{2}}\right] .
$$

The pressure and density are determined by assuming an isentropic initial flow condition:

$$
\rho(\boldsymbol{x})=\rho_{\infty}\left[1-\frac{\gamma-1}{2} \psi^{2}(\boldsymbol{x})\right]^{\frac{1}{\gamma-1}}, \quad p(\boldsymbol{x})=p_{\infty}\left(\frac{\rho}{\rho_{\infty}}\right)^{\gamma} .
$$

The subscript " $\infty$ " denotes the free-stream condition. The free parameters $\sigma$ and $\epsilon$ in Eq. (6) determine the size and strength of the vortex. In the current work, $\sigma=44.25$ is fixed and $\epsilon=\epsilon_{0}=0.0377$ is used as standard in Cases 1, 4 and 5 (see Table 1). Other values, $\epsilon=\epsilon_{0} / 2, \epsilon_{0} / 4$ in Cases 2 and 3. Higher vortex strengths $\epsilon=2 \epsilon_{0}, 4 \epsilon_{0}$ and $6 \epsilon_{0}$ are also considered in the viscous cases. The vortex induced velocity reaches $2.5 \%$ of the free-stream velocity (i.e. $\left.|v|_{\max } / u_{\infty}=0.025\right)$ with the default vortex strength $\left(\epsilon=\epsilon_{0}\right)$ used, and it changes proportionally when a different vortex strength is chosen.

The size (radius) of the vortex is approximately $8 \%$ of the aerofoil chord, defined by the locations where the induced velocity drops down to $1 \%$ of the maximum value. This vortex size approximately corresponds to $10 \delta_{\mathrm{TE}}$ where $\delta_{\mathrm{TE}}$ is the thickness of the laminar boundary layer at the aerofoil TE in the viscous cases to be discussed in Section 5 . Also, the vortex core radius (measured from the locations of the maximum induced velocity) is around $3 \delta_{\mathrm{TE}}$. Due to the adequate vortex size, there is a sufficient time gap between the LE and TE interactions, which facilitates the signal processing of the results. Also, the vortex contains a sufficiently wide range of frequency components due to the Gaussian shape function.

The vortex generates a clockwise circulation viewed from the $x y$-plane where the vortex travels from left to right. Therefore the aerofoil faces a downwash first followed by an upwash during the course of interaction with the vortex. The simulations are run for 15 non-dimensional time units $\left(15 t^{*}=15 t a_{\infty} / L_{c}\right)$ for the inviscid cases and $20 t^{*}$ for the viscous cases (with a fully-developed base flow set as the initial condition). By the end of the simulation, the initial vortex and any smaller-scale vortical structures created 
as a result of its interaction with the aerofoil have reached at least $2 L_{c}$ downstream of the trailing edge. Also the entirety of the acoustic waves generated by the primary and secondary sources have travelled past the observer location that is $5 L_{c}$ away in the vertical direction from the centre of the aerofoil, i.e. $\boldsymbol{x}_{\mathrm{o}} / L_{c}=(0,5,0)$.

\subsection{Definition of variables for statistical analysis}

Data processing and analysis are carried out upon the completion of each simulation. The main property required in this study is the power spectral density (PSD) function of pressure fluctuations on the aerofoil surface and at the far-field observer location. The far-field (acoustic) pressure and the surface (wall) pressure loading are defined as:

$$
p_{a}(\boldsymbol{x}, t)=p(\boldsymbol{x}, t)-\bar{p}(\boldsymbol{x}),
$$

where $\bar{p}(\boldsymbol{x})$ is the time averaged pressure, and

$$
\Delta p_{w}(\boldsymbol{x}, t)=\lim _{y \rightarrow 0^{+}} p(\boldsymbol{x}, t)-\lim _{y \rightarrow 0^{-}} p(\boldsymbol{x}, t),
$$

where superscripts ' $y \rightarrow 0^{+}$' and ' $y \rightarrow 0^{-}$' indicate the upper and lower surfaces of the zero-thickness aerofoil, respectively. Following the definitions used in Goldstein [51], the PSD functions of the pressure fluctuations (based on frequency and one-sided) are then calculated by:

$$
\begin{gathered}
S_{p p a}(\boldsymbol{x}, f)=\lim _{T \rightarrow \infty} \frac{P_{a}(\boldsymbol{x}, f, T) P_{a}^{*}(\boldsymbol{x}, f, T)}{T}, \\
S_{p p w}(\boldsymbol{x}, f)=\lim _{T \rightarrow \infty} \frac{\Delta P_{w}(\boldsymbol{x}, f, T) \Delta P_{w}^{*}(\boldsymbol{x}, f, T)}{T},
\end{gathered}
$$

where $P_{a}$ and $\Delta P_{w}$ are an approximate Fourier transform of $p_{a}$ and $\Delta p_{w}$, respectively, based on the following definition:

$$
\begin{gathered}
P_{a}(\boldsymbol{x}, f, T)=\int_{-T}^{T} p_{a}(\boldsymbol{x}, t) e^{2 \pi \mathrm{i} f t} \mathrm{~d} t, \\
\Delta P_{w}(\boldsymbol{x}, f, T)=\int_{-T}^{T} \Delta p_{w}(\boldsymbol{x}, t) e^{2 \pi \mathrm{i} f t} \mathrm{~d} t,
\end{gathered}
$$

and, ' $*$ ' denotes a complex conjugate. In the above equations, $T$ represents the half length of the time signals used for the approximate Fourier transform. The same definitions are also used for the Fourier transform of vorticity later in this paper.

\section{Secondary noise sources in aerofoil-vortex interaction}

This section provides a detailed observation on the secondary noise sources based on inviscid solutions which can be discussed in comparison with the previous theoretical solutions. The main focus in this investigation is on the extent of the contribution of TEVS (trailing-edge vortical scattering) to the radiated sound as suggested in Section 1. One of the advantages of using the inviscid simulation set-up is that it is possible to cleanly separate each source component (LEVS, TEVS and ABS) from the total (although this is not the case in the viscous cases - to be detailed in Section 5). The separation procedure is as follows. First, the primary solution for LEVS (Fig. 2 $2 a$ ) is obtained by using a semi-infinite-chord aerofoil. Secondly, the total solution that includes all sources 
(a)

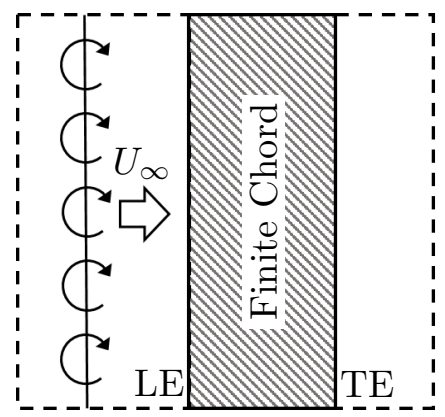

(b)

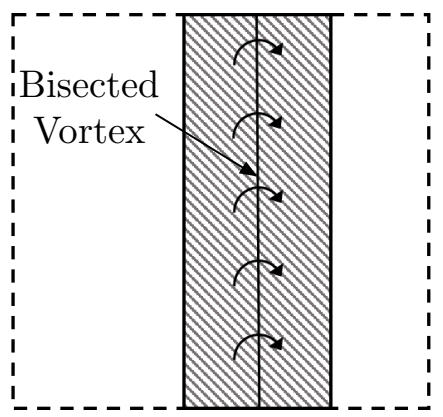

(c)

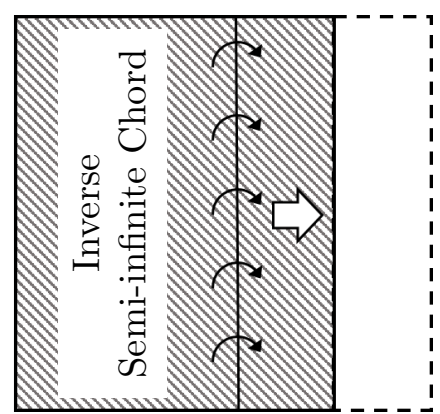

Figure 3: An auxiliary simulation procedure in order to extract the pure inviscid TEVS solution: $(a)$ first, obtain an instantaneous solution with a finite-chord aerofoil at the time when the impinging vortex (bisected at the LE) arrives at the mid-chord location $(x=0)$; $(b)$ reinstate the free-stream condition outside a perimeter of $r / L_{c}=1 / 4$ from $x=0$, which effectively removes the acoustic waves (LEVS and ABS) that have all radiated outside the perimeter by that time but maintain the bisected vortices inside the perimeter (on both the upper and lower sides of the aerofoil); (c) extend the slip-wall boundary condition upstream to remove the LE and restart the simulation.

(Fig. $2 b$ ) is obtained by using a finite-chord aerofoil. By subtracting the primary solution from the total, the secondary solution is filtered out as follows:

$$
p_{\text {secondary }}(\boldsymbol{x}, t)=p_{\text {total }}(\boldsymbol{x}, t)-p_{\text {primary }}(\boldsymbol{x}, t) \quad \text { where } \quad p_{\text {primary }}=p_{\text {LEVS }} .
$$

This process is valid because the diameter of the impinging vortex is sufficiently smaller than the aerofoil chord $\left(0.16 L_{c}\right)$ and therefore the primary source remains identical regardless of the finite and semi-infinite chords. Thirdly, an auxiliary simulation is carried out in order to extracts a pure TEVS solution, i.e. $p_{\text {TEVS }}(\boldsymbol{x}, t)$. The auxiliary simulation is based on a selected portion of the total solution set as its initial condition. The details of the auxiliary simulation are described in Fig. 3. Once the pure TEVS solution is obtained from the auxiliary simulation, the pure ABS solution is

$$
p_{\mathrm{ABS}}(\boldsymbol{x}, t)=p_{\text {secondary }}(\boldsymbol{x}, t)-p_{\mathrm{TEVS}}(\boldsymbol{x}, t) .
$$

In this fashion, the three individual solutions are made available to facilitate the investigation.

\subsection{Initial findings}

The acoustic pressure data obtained at the far field as a result of the current inviscid simulations (Case 1 in Table 1) are plotted in Fig. 4. The semi-infinite aerofoil offers the pure primary solution (LEVS) and the finite aerofoil generates all sources (both the primary and secondary). One evident observation that can be made in Fig. $4(a)$ is that the secondary sources make significant changes in the sound time signal from $t^{*}=8$ onward. Fig. $4(b)$ shows the differences in the frequency domain. First, the sound power spectrum becomes noticeably oscillatory with the secondary sources included. This is due to phase interferences between the three different noise sources (LEVS, TEVS and ABS), i.e. time differences in the source emission and the acoustic travelling to the observer. Secondly, there is a drastic change in the slope of the spectra at high frequencies $\left(f^{*}>5\right)$ when the secondary sources are involved. The high-frequency behaviour of the secondary sources is one of the main points of discussion in this paper. 

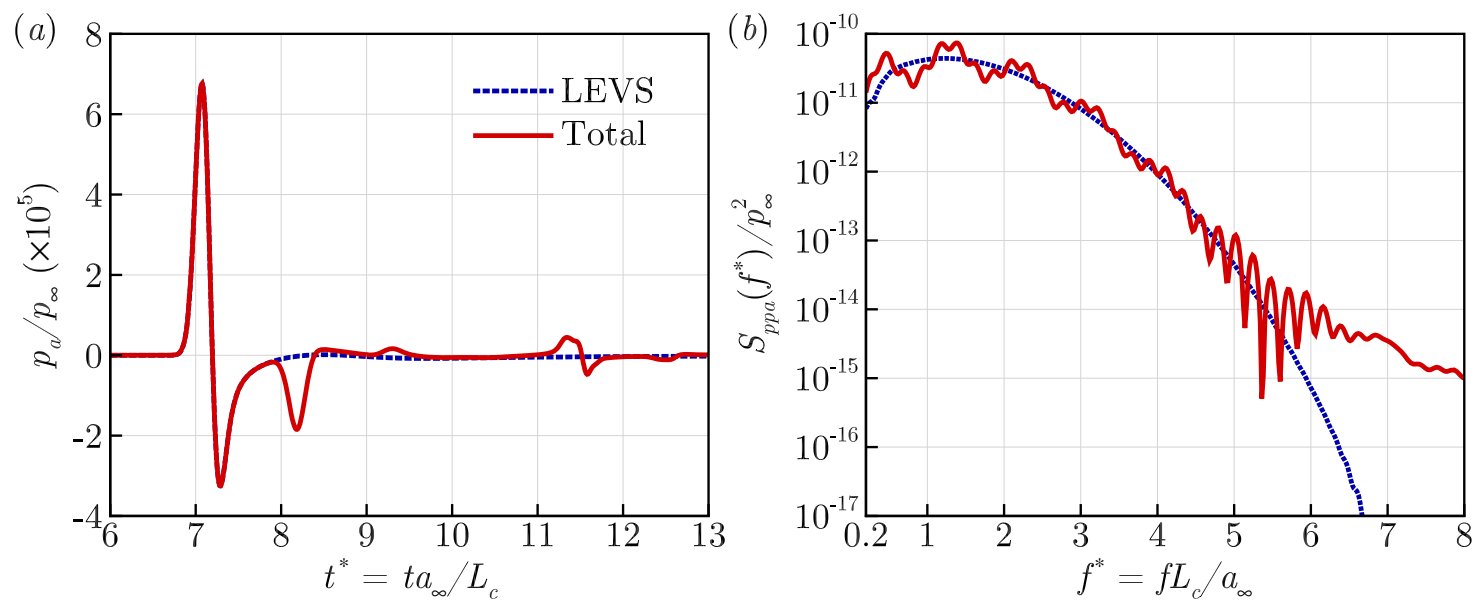

Figure 4: Acoustic pressure data obtained at an observer location from the current inviscid simulations (Case 1 in Table 1 with a finite and a semi-infinite aerofoil, where the former includes all noise sources and the latter contains the primary LEVS only): (a) time signals of $p_{a}\left(\boldsymbol{x}_{\mathrm{o}}, t\right) / p_{\infty}$ where $\boldsymbol{x}_{\circ} / L_{c}=(0,5)$; (b) the corresponding power spectra; LEVS stands for leading-edge vortical scattering.
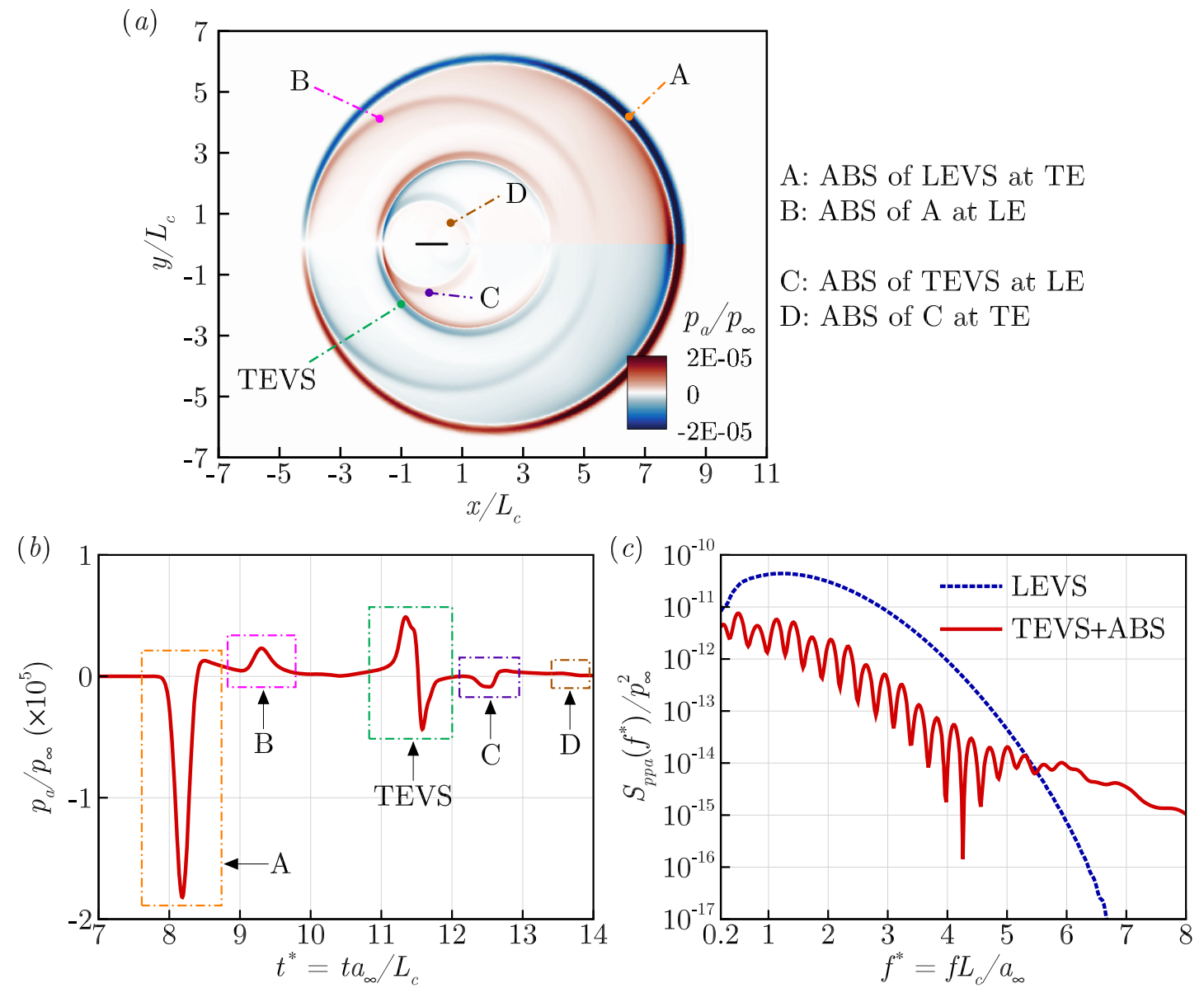

Figure 5: Acoustic pressure generated by the secondary sources (with the primary source excluded) obtained by using Eq. 14p: (a) an instantaneous contour plot of the acoustic pressure $p_{a} / p_{\infty}$ at the time of the impinged vortex reaching $x / L_{c}=1.16 ;(b)$ the time signal of $p_{a} / p_{\infty}$ obtained at the observer location $\boldsymbol{x}_{\mathrm{\circ}} / L_{c}=(0,5) ;(c)$ the corresponding power spectrum compared to that of the primary source; the results from Case 1 in Table 1 are displayed here; the acronyms are defined in Fig. 2 (as well as in the text). 


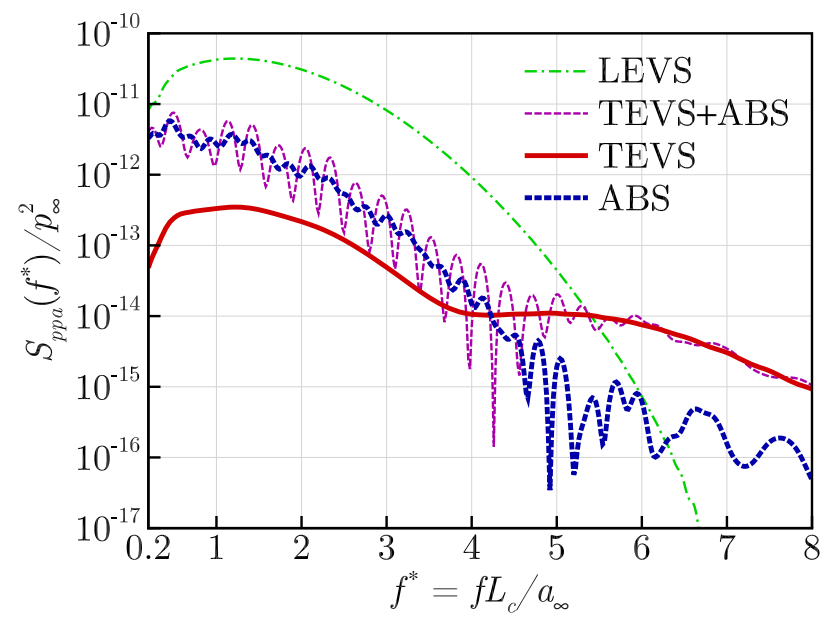

Figure 6: Acoustic power spectra for the individual secondary source components; the one denoted by TEVS+ABS represents the combined secondary solution presented in Fig. $5(c)$.

As explained earlier, via Eq. (14), the secondary solution containing the TEVS and ABS is obtained and shown in Fig. 5. Five sub-components of the secondary solution are detailed in Figs. $5(a)$ and $(b)$, i.e. 1) the first sub-component (labelled A) is due to the ABS (acoustic backscattering) of the initial wave that was created by the primary source (LEVS) shown in Fig. 2(a);2) B is the subsequent ABS of A; 3) TEVS is the vortical scattering at the TE as defined earlier; 4) $\mathrm{C}$ is the ABS of the TEVS; and, 5) D is the subsequent $\mathrm{ABS}$ of $\mathrm{C}$. In this paper, the $\mathrm{ABS}$ represents the sum of $\mathrm{A}, \mathrm{B}, \mathrm{C}$ and $\mathrm{D}$, where the contribution of $\mathrm{D}$ is insignificant compared to the others.

It is confirmed in Fig. 5( $(c)$ that the secondary sources are responsible for the highfrequency noise $\left(f^{*}>5\right)$ whereas the low-to-medium frequencies $\left(f^{*}<5\right)$ are governed by the primary source as expected. It should also be noted that the spectral oscillations in the secondary solution disappear in the high-frequency range indicating that the phase interference between the sub-components (TEVS and A to D mentioned above) becomes insignificant. In other words one of the sub-components starts to dominate over the others from about $f^{*} \approx 5$ and therefore there is no longer a meaningful competition taking place between them after the critical frequency. The next logical step in this investigation is to figure out which one of them is truly responsible for the strong high-frequency activities.

\subsection{Dominance of TEVS at high frequencies}

In order to find an answer to the question raised above, the secondary solution is further split into two (one for the TEVS and the other for the ABS) through the procedure explained in Fig. 3 and Eq. (15). The resulting acoustic power spectra obtained at the observer location $\boldsymbol{x}_{\mathrm{o}} / L_{c}=(0,5)$ are presented in Fig. 6 for each of the split solutions. The figure clearly shows that the TEVS is the dominating secondary source component in the high-frequency range $\left(f^{*}>5\right)$. It also reveals that the TEVS even exceeds the primary source (LEVS) there. On another note, it is shown that the spectral oscillations in the combined TEVS and ABS case intensify when the amplitudes of the TEVS and ABS are similar, but weaken when one of them is superior to the other. This is a strong indication of the phase relationship existing between the two secondary sources. The high-frequency dominance of the TEVS is found consistent over a wide range of observer angles.

Fig. 7 shows the directivity patterns of $S_{p p a}\left(f^{*}\right) / p_{\infty}^{2}$ for two different frequencies: $f^{*}=2$ and 6 . With the semi-infinite plates, the pure LEVS and TEVS solutions produce 

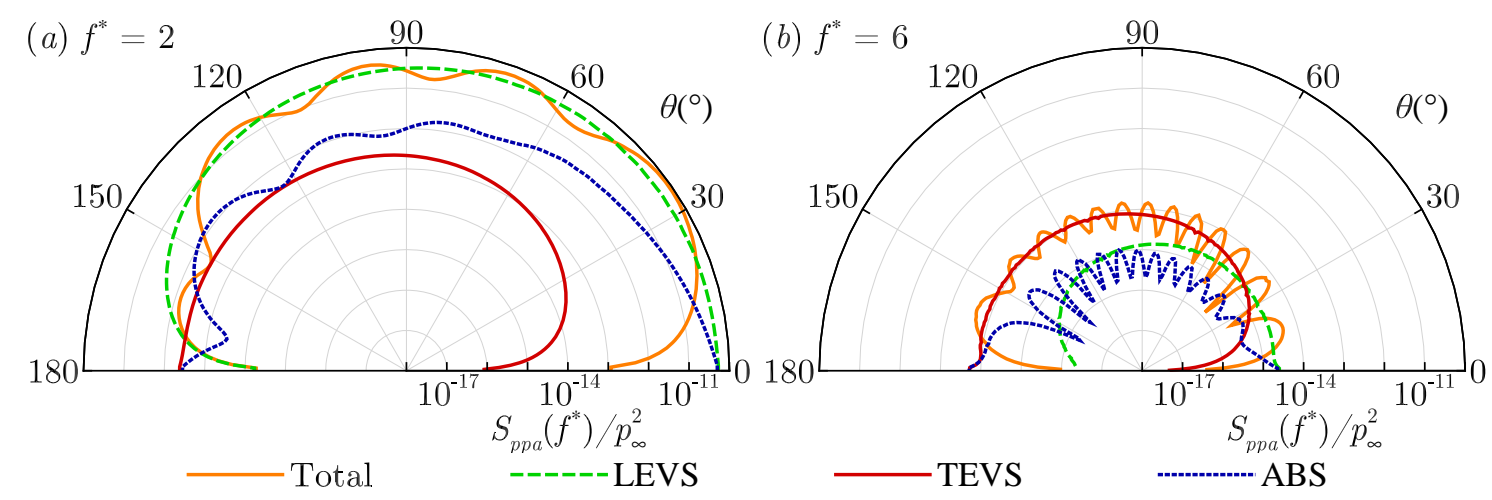

Figure 7: Directivity profiles of $S_{p p a}\left(f^{*}\right) / p_{\infty}^{2}$ (on a logarithmic scale) at two different frequencies: $(a)$ $f^{*}=2$ and $(b) f^{*}=6$, around a semi-circle of $\boldsymbol{x}_{\mathrm{o}} / L_{c}=(5 \cos \theta, 5 \sin \theta)$, obtained from each individual noise source compared to the total; lower half planes are not repeated due to symmetry.
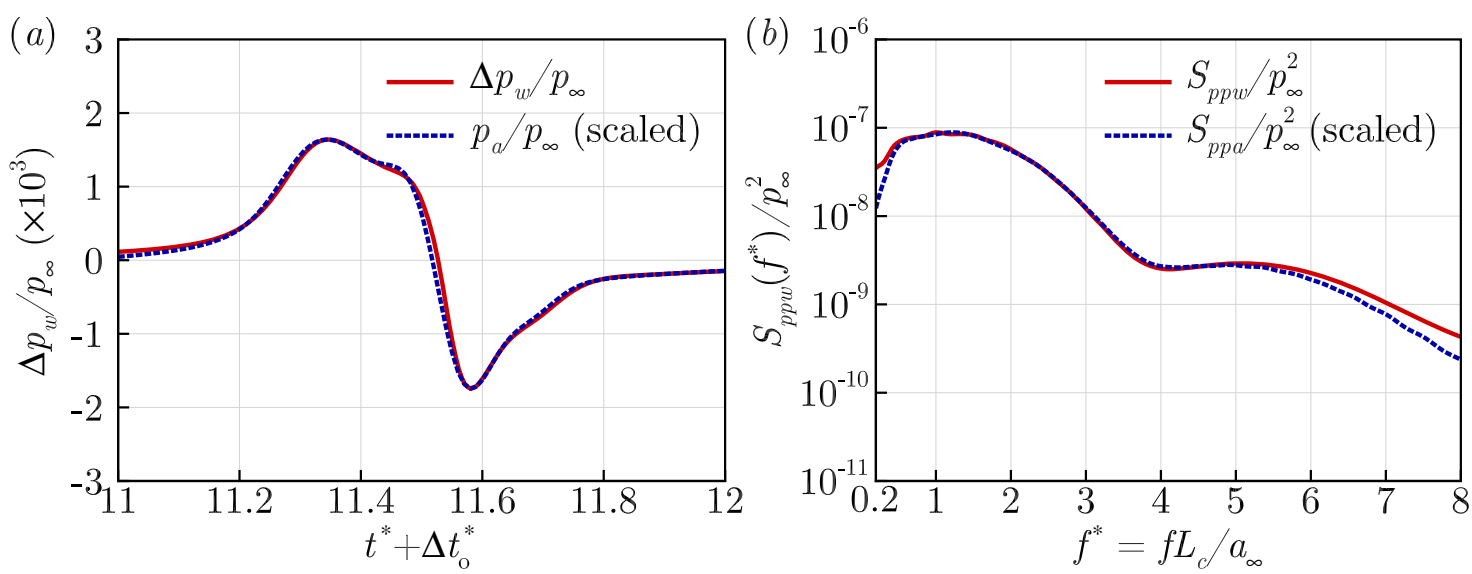

Figure 8: Wall pressure loading fluctuations at the TE $\left(\Delta p_{w}\right)$ compared with the far-field acoustic pressure $\left(p_{a}\right)$, obtained from the pure TEVS solution, $p_{\text {TEVS }}(\boldsymbol{x}, t)-$ see Fig. 3 ( $\left.a\right)$ the time signals where $\Delta t_{\mathrm{o}}^{*}$ represents the retarded time for the radiated sound to arrive at the observer location; $(b)$ the corresponding power spectra; the far-field acoustic pressure is scaled up to the level of the wall pressure loading fluctuations.

cardioid directivity patterns as expected [52]. Meanwhile, the total and ABS solutions contain lobes caused by interference effects due to the finite chord length. At the low frequency, the LEVS dictates in all directions, followed by the ABS. However, at the high frequency, the TEVS is the strongest source in most directions except the narrow downstream region $\left(\theta \leq 30^{\circ}\right)$ where the LEVS maintains its strength.

It is worth checking that the high-frequency dominance of the TEVS noise is genuine and free from any numerical artefact potentially caused during the process of splitting the secondary solutions implemented earlier. For this purpose, the source (wall pressure loading fluctuations) $\Delta p_{w}(t)$ obtained at one grid point upstream of the TE is compared with the radiated sound pressure $p_{a}(t)$. Fig. 8 shows the time signals and the corresponding power spectra of both quantities, compared with each other. The figure reveals that the source and the radiated noise are in perfect correlation across all frequencies. This evidences that the high-frequency characteristics of the TEVS noise are properly captured in the current numerical solution without spurious numerical artefacts involved. It is necessary though to understand the details of the vortex dynamics and evolution processes that have taken place along the aerofoil surface before reaching the TE - to be followed in Section 4 . 

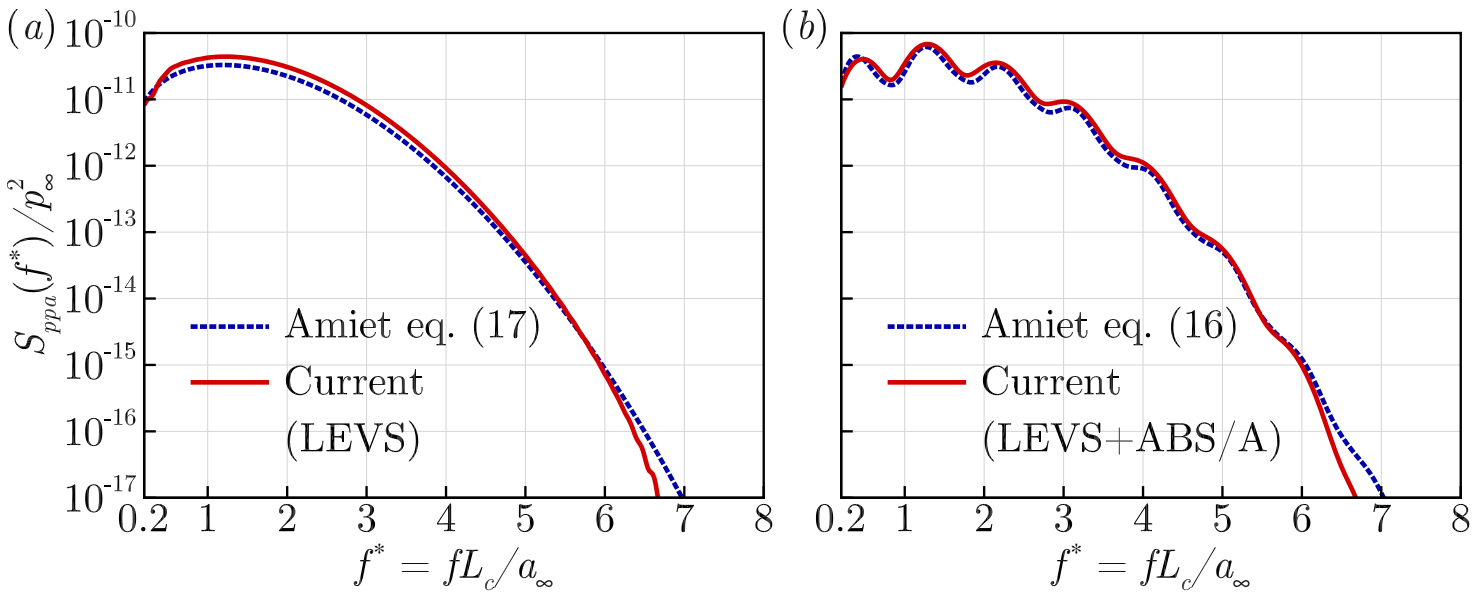

Figure 9: Amiet's model predictions of the acoustic power spectra at $\boldsymbol{x}_{\circ} / L_{c}=(0,5)$ given by Eqs. 16 ) and 17) compared with the current simulation results, with respect to two different source scenarios: (a) LEVS only and (b) LEVS and ABS/A combined; the velocity power spectrum $\left(\Phi_{v v}\right)$ required in the Amiet's models is given by Eqs. (5) and (6).

\subsection{Comparison with analytical predictions}

An analytical prediction is also attempted in order to double check the validity of the current numerical solutions. One of the most popular frequency-based analytical models for aerofoil noise prediction was proposed by Amiet [1, 53. The Amiet's model is essentially split into two independent models where one is for leading-edge noise due to impinging free-stream turbulence and the other for trailing-edge self-noise due to boundary-layer turbulence. The first one [1] naturally has a direct relevance to the current work. Blandeau et al. 54] recently derived a two-dimensional version (for an infinite span) of the original model which can directly be compared to the current results. The two-dimensional model is written as

$$
S_{\text {ppa-Amiet } 1}\left(k_{x}\right)=\frac{\pi k_{x} M_{\infty} \rho_{\infty}^{2} u_{\infty}^{2} L_{c}^{2} \sin ^{2} \theta}{8 r_{\mathrm{o}} A^{3}(\theta)} \Phi_{v v}\left(k_{x}\right)\left|\mathcal{L}\left(k_{x}\right)\right|^{2},
$$

where $k_{x}=2 \pi f / u_{\infty} ; A(\theta)=\left(1-M_{\infty}^{2} \sin ^{2} \theta\right)^{1 / 2} ; r_{\mathrm{o}}$ is the observer distance from the midchord of the aerofoil; $\Phi_{v v}\left(k_{x}\right)$ is the PSD of the vertical velocity fluctuations impinging on the LE of the aerofoil (which is obtainable from Eq. (5)); and, $\mathcal{L}\left(k_{x}\right)$ describes the unsteady loading on the aerofoil - see [54] for full details. This model accommodates the primary source (LEVS) and the first acoustic backscattering component (ABS/A in Fig. 5). However, this means that the model omits the TEVS and the rest of the acoustic backscattering components (ABS/B, C and D). In this paper we apply a semi-infinitechord approximation $\left(L_{c} \rightarrow \infty\right)$ to Eq. (16) and remove the acoustic backscattering term in $\mathcal{L}\left(k_{x}\right)$ in order to obtain the pure LEVS solution (after some algebraic manipulations):

$$
S_{p p a-\operatorname{Amiet1-LEVS}}\left(k_{x}\right)=\frac{\rho_{\infty}^{2} u_{\infty}^{2}\left(1-M_{\infty}\right) \sin ^{2} \theta}{2 \pi r_{\mathrm{o}} k_{x} A(\theta)^{2}[A(\theta)-\cos \theta]} \Phi_{v v}\left(k_{x}\right) .
$$

A comparison of the analytical predictions and the current simulation results is provided in Fig. 9. It is shown that there is a very good agreement between them (except at the very high frequencies), which confirms the validity of both approaches.

In the absence of a prediction model for the TEVS noise at present, Amiet's second prediction model [53] developed for TE scattering of boundary-layer turbulence (self-noise) and its acoustic backscattering at the LE could be used as an alternative. This is also 

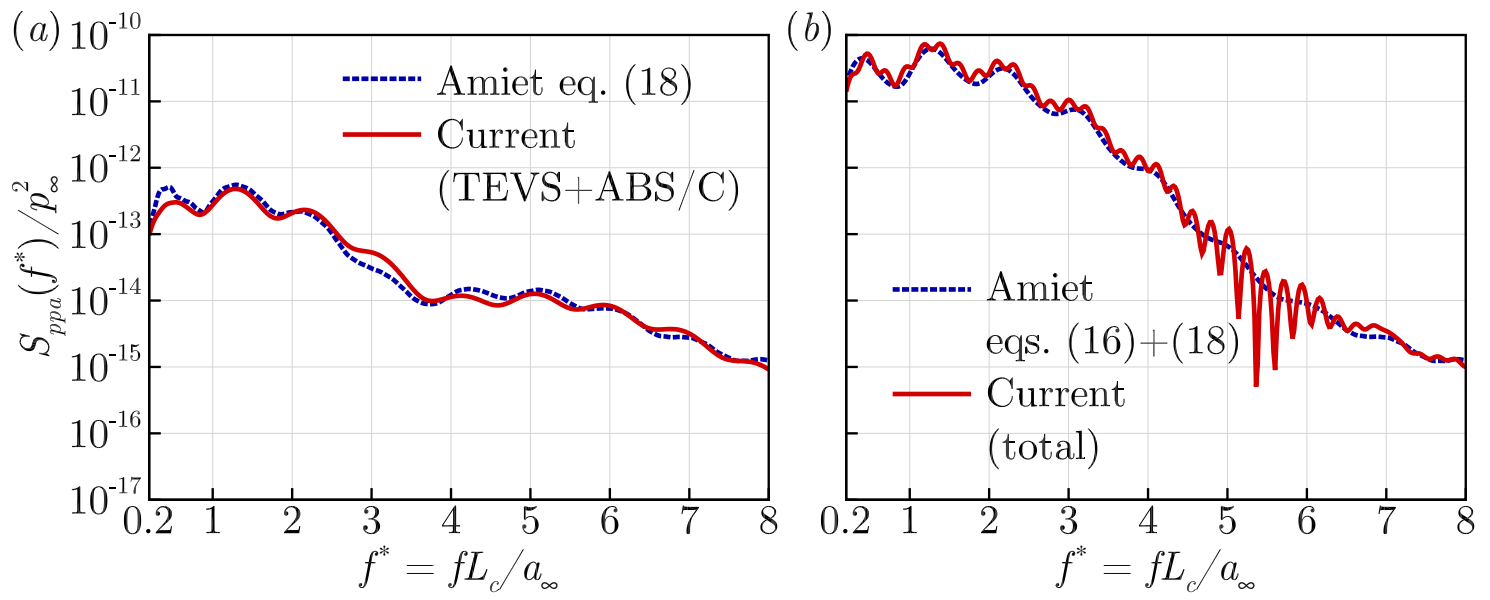

Figure 10: Amiet's model predictions of the acoustic power spectra at $\boldsymbol{x}_{\mathrm{\circ}} / L_{c}=(0,5)$ : $(a)$ by using Eq. 18 for TEVS and ABS/C, and (b) by adding Eqs. (16) and 18 to represent a combination of LEVS, TEVS, ABS/A and C (without complete phase interactions between them); the current simulation result in (b) includes all noise sources (LEVS, TEVS and ABS/A to D) and their phase interactions; the $S_{Q Q}$ term required in Eq. 18 is calculated by using the simulation data $\left(\Delta p_{w}\right)$ consisting of TEVS and ABS/C.

achievable by using Roger and Moreau [55]. However, this approach requires wall pressure data - similarly to Ffowcs Williams and Hawkings [56 - which are not directly available from the given upstream turbulence, hence the limitations. The two-dimensional version (for an infinite span) of the self-noise model has been derived by Sandberg and Sandham [57] as

$$
\left.\begin{array}{c}
S_{\text {ppa-Amiet } 2}\left(k_{x}\right)=-\frac{\beta^{2} \mu_{0}^{2}}{16}\left(y_{\mathrm{TE}}-y_{\mathrm{o}}\right)^{2} \iint \frac{S_{Q Q}\left(x_{1}, x_{2}, k_{x}\right)}{R\left(x_{1}\right) R\left(x_{2}\right)} \times \\
H_{1}^{(2)}\left(S\left(x_{1}\right)\right) H_{1}^{(1)}\left(S\left(x_{2}\right)\right) \mathrm{d} x_{1} \mathrm{~d} x_{2}, \\
=\sqrt{\left(x-x_{\mathrm{o}}\right)^{2}+\beta^{2}\left(y_{\mathrm{TE}}-y_{\mathrm{o}}\right)^{2}}, \quad S(x)=\mu_{0}\left[M_{\infty}\left(x-x_{\mathrm{o}}\right)+R(x)\right]
\end{array}\right\}
$$

where $\mu_{0}=M_{\infty} L_{c} k_{x} /\left(2 \beta^{2}\right) ; \beta=\left(1-M_{\infty}^{2}\right)^{1 / 2} ; H_{1}^{(1)}$ and $H_{1}^{(2)}$ are the first-order Hankel functions of the first and second kinds, respectively; and, $S_{Q Q}$ is the cross-PSD of $\Delta p_{w}$ over the aerofoil surface. This model is able to accommodate the TEVS and ABS/C (via $S_{Q Q}$ ) for this study although they are not produced by a turbulent boundary layer. The calculated values of Eq. (18) are presented in Fig. 10 compared with the current simulation data. Again it is demonstrated that the analytical predictions and the simulation data agree very well each other. This result re-confirms the validity of the current numerical solutions particularly with regard to the high-frequency dominance of the TEVS noise.

\section{High-frequency TEVS mechanisms}

The next question is then what mechanism drives the high-frequency dominance of the TEVS noise. As mentioned earlier in the paper, Abou-Hussein et al. [30], Howe [32], Manela [34] suggested that nonlinear effects were responsible for the noise generated at the TE in the interaction of an aerofoil with a vortex. However, the earlier analytical work did not reveal tangible information about the frequency contents of the nonlinear effects that might have been relevant to the high-frequency TEVS event. Also, it is naturally anticipated that the "distributed" vortex used in this work (as opposed to the singular point vortex used in the analytical work) will behave differently during the course 

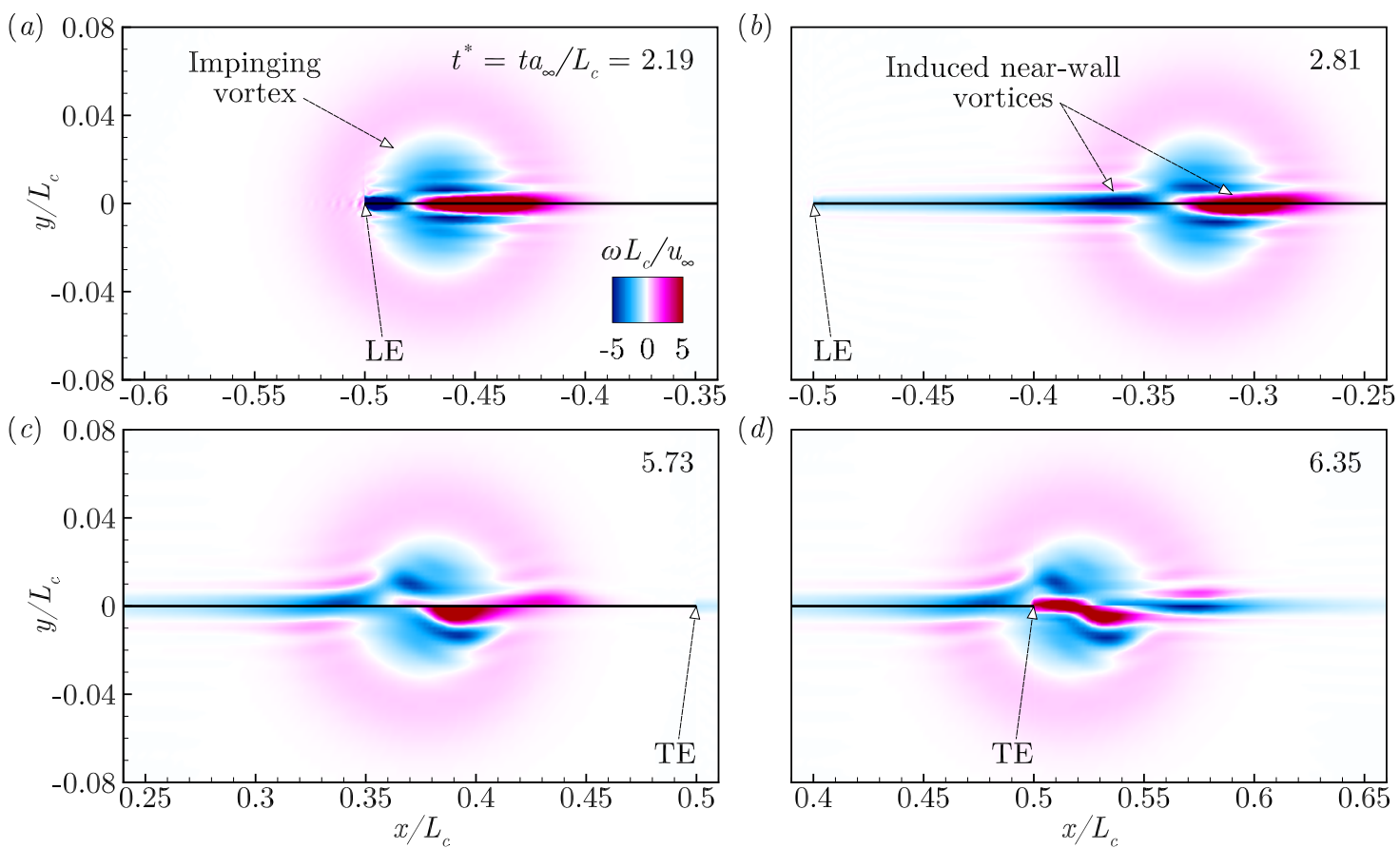

Figure 11: Snapshots of vorticity $\left(\omega L_{c} / u_{\infty}\right)$ taken at four different points in time: $(a) t^{*}=t a_{\infty} / L_{c}=$ 2.19, (b) 2.81, (c) 5.73 and (d) 6.35, where the first two are near the LE and the later two near the TE; Case 1 in Table 1 (with a finite aerofoil) is presented in this figure.

of interaction with the aerofoil. In this section, a detailed investigation is made in order to find the origin of the high-frequency dominance of the TEVS noise identified in the current study.

\subsection{Induced near-wall vortices}

In order to understand the TEVS mechanism, it is necessary to view how the flow field changes around the aerofoil during its interaction with the impinging vortex. Fig. 11 shows vorticity contour plots $(\omega=\partial v / \partial x-\partial u / \partial y)$ taken at four different points in time (two when the impinging vortex is near the LE and the other two near the TE). It is shown in the figure that the impinging vortex is bisected by the LE and more importantly there are additional vortical structures induced at and around the LE as a result of the interaction. The induced vortices travel with the initial impinging vortex all the way to the TE and they remain very close to the wall throughout the entire journey. It is found that the induced near-wall vortices have a significantly higher intensity compared to the initial impinging vortex. Also, it is noticeable that the induced near-wall vortices on the upper side make a different evolution to those on the lower side when they arrive at the TE. It is rather obvious to see the asymmetric evolution of the vortices due to the antisymmetric vorticity distribution from the mirror/image point of view across the wall. The near-wall vortices are highlighted in Fig. 12 by subtracting the background impinging vortex, i.e. plotting $\Delta \omega(\boldsymbol{x}, t)=\omega(\boldsymbol{x}, t)-\omega_{\text {free-field }}(\boldsymbol{x}, t)$ where $\omega_{\text {free-field }}(\boldsymbol{x}, t)$ is a free-field solution obtained without the aerofoil. It is shown in the figure that the near-wall vortices exhibit significantly more irregular patterns broken into smaller scales when they arrive at TE, compared to them being in the vicinity of the LE. This indicates the level of high-frequency components increasing as the vortices travel downstream.

The frequency contents of the induced near-wall vortices probed at various positions 

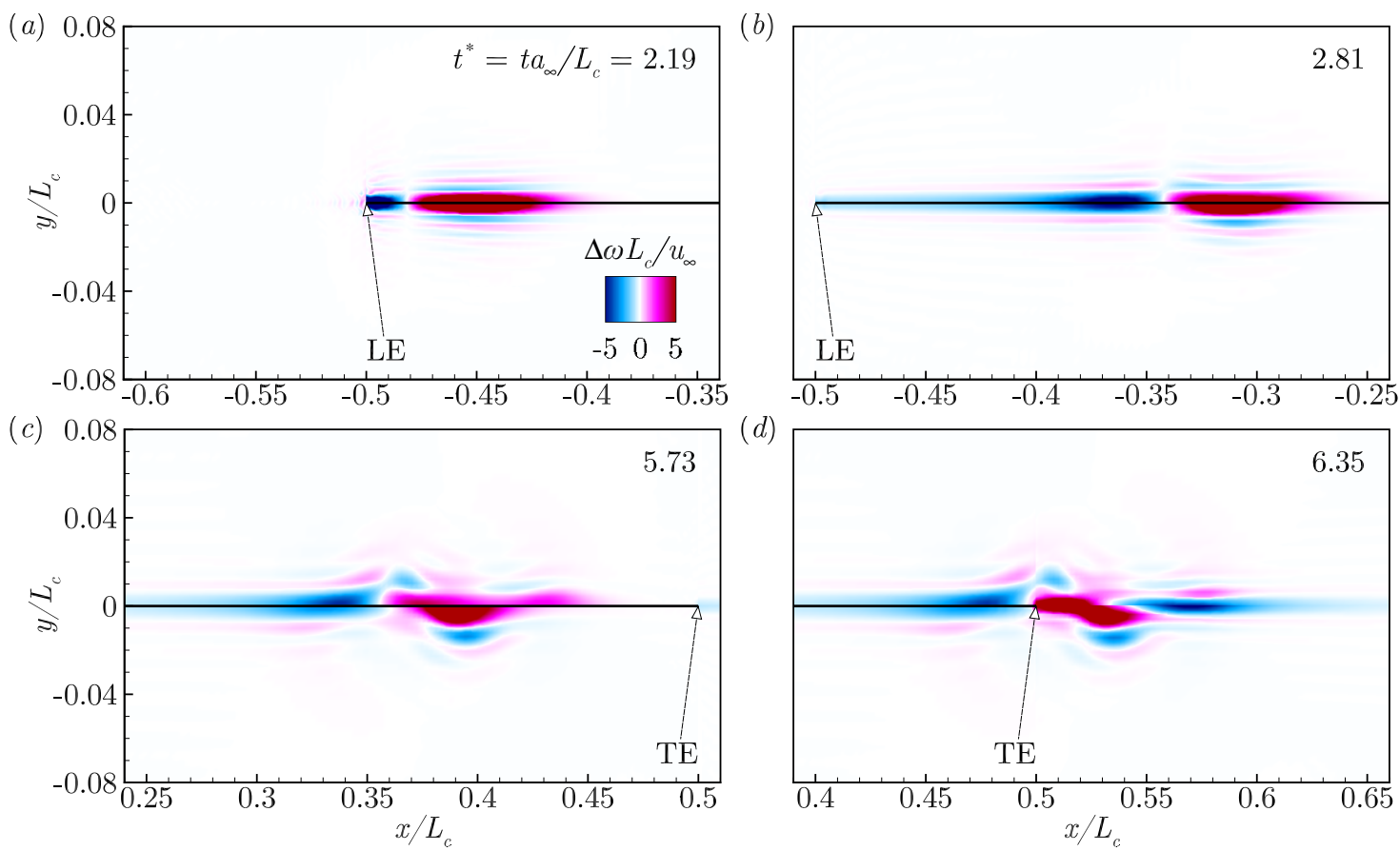

Figure 12: A reproduction of Fig. 11 after subtracting the background impinging vortex (the free-field solution without the aerofoil), i.e. $\Delta \omega(\boldsymbol{x}, t)=\omega(\boldsymbol{x}, t)-\omega_{\text {free-field }}(\boldsymbol{x}, t)$; the figure shows the additional vorticity distribution induced due to the interaction between the impinging vortex and the aerofoil.
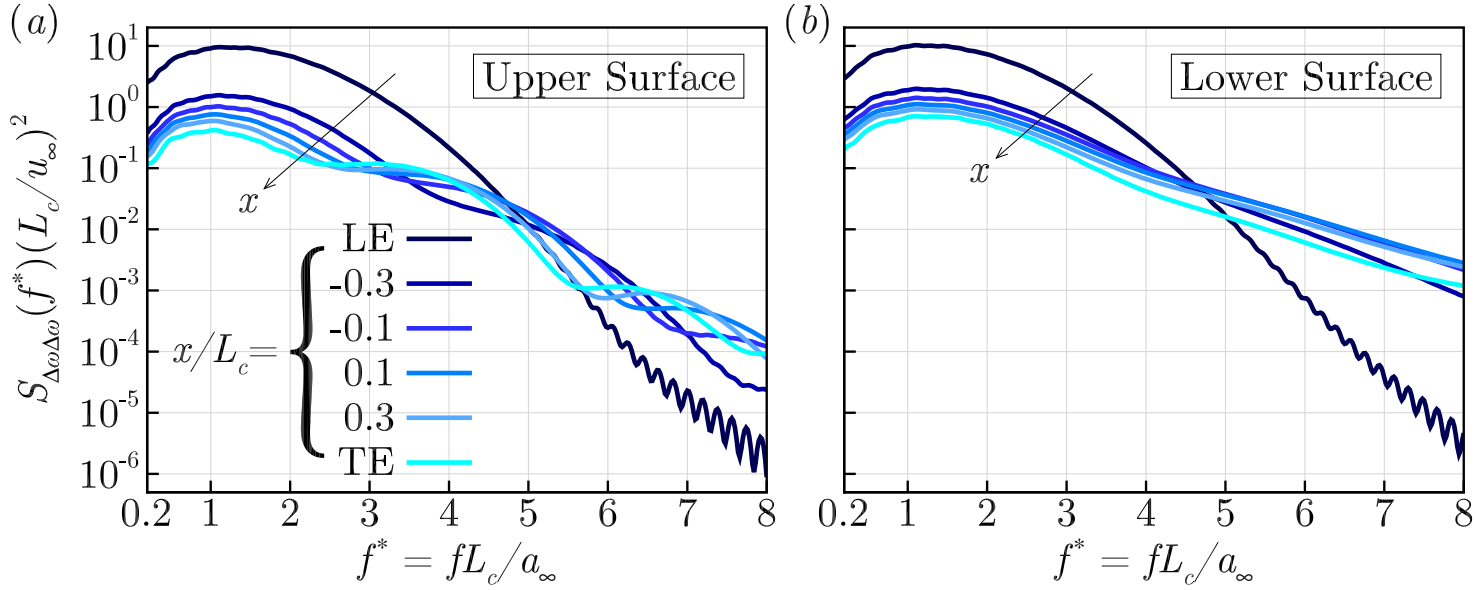

Figure 13: Power spectra of the time signals of the induced near-wall vorticity $\left(\Delta \omega L_{c} / u_{\infty}\right.$ as shown in Fig. 12 where $\Delta \omega=\omega-\omega_{\text {free-field }}$ ) obtained at various locations on the aerofoil: ( $a$ ) upper and $(b)$ lower surfaces; the LE and TE points denoted in this figure are one grid cell recessed onto the aerofoil surface from the actual LE and TE points, i.e. $x_{\mathrm{LE}}+\Delta x$ and $x_{\mathrm{TE}}-\Delta x$. 

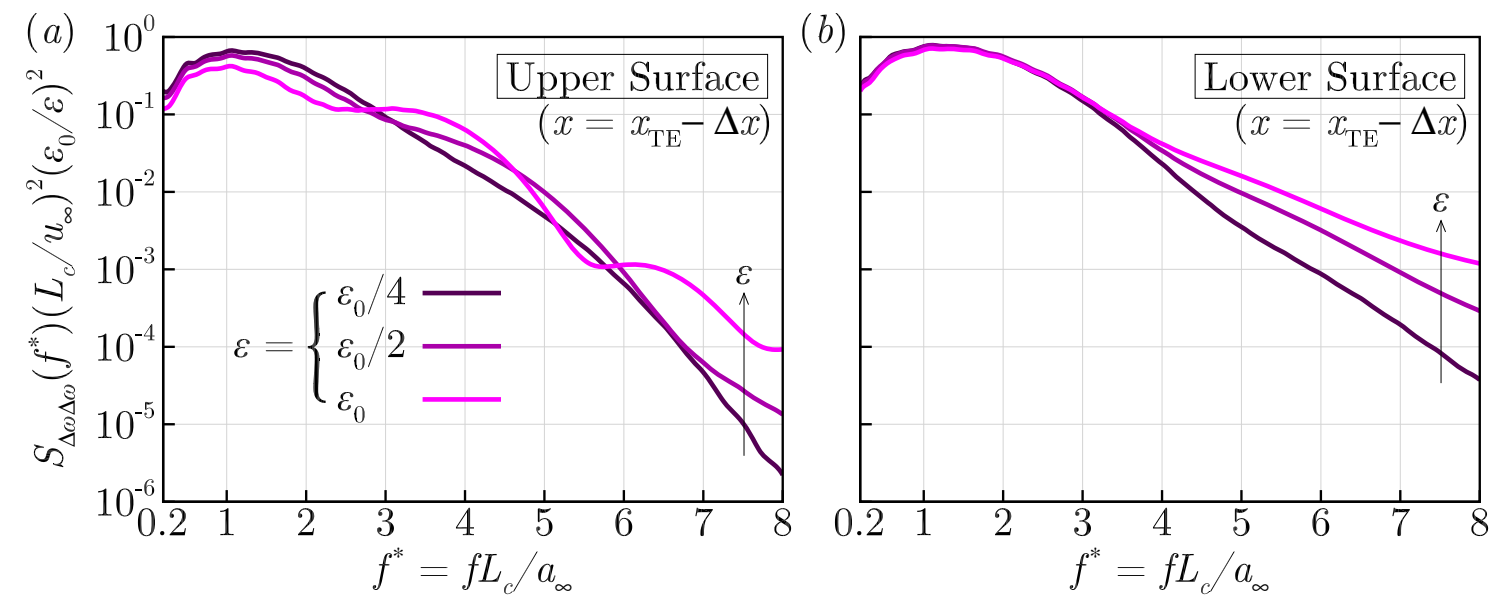

Figure 14: Power spectra of the time signals of the induced near-wall vorticity (same as those shown in Fig. 13 obtained from three different strengths of the impinging vortex: $\epsilon=\epsilon_{0}, \epsilon_{0} / 2$ and $\epsilon_{0} / 4$ where $\epsilon_{0}=0.0377-$ see Eq. 6]; the vorticity is re-scaled by $\epsilon_{0} / \epsilon$ for comparison purposes.
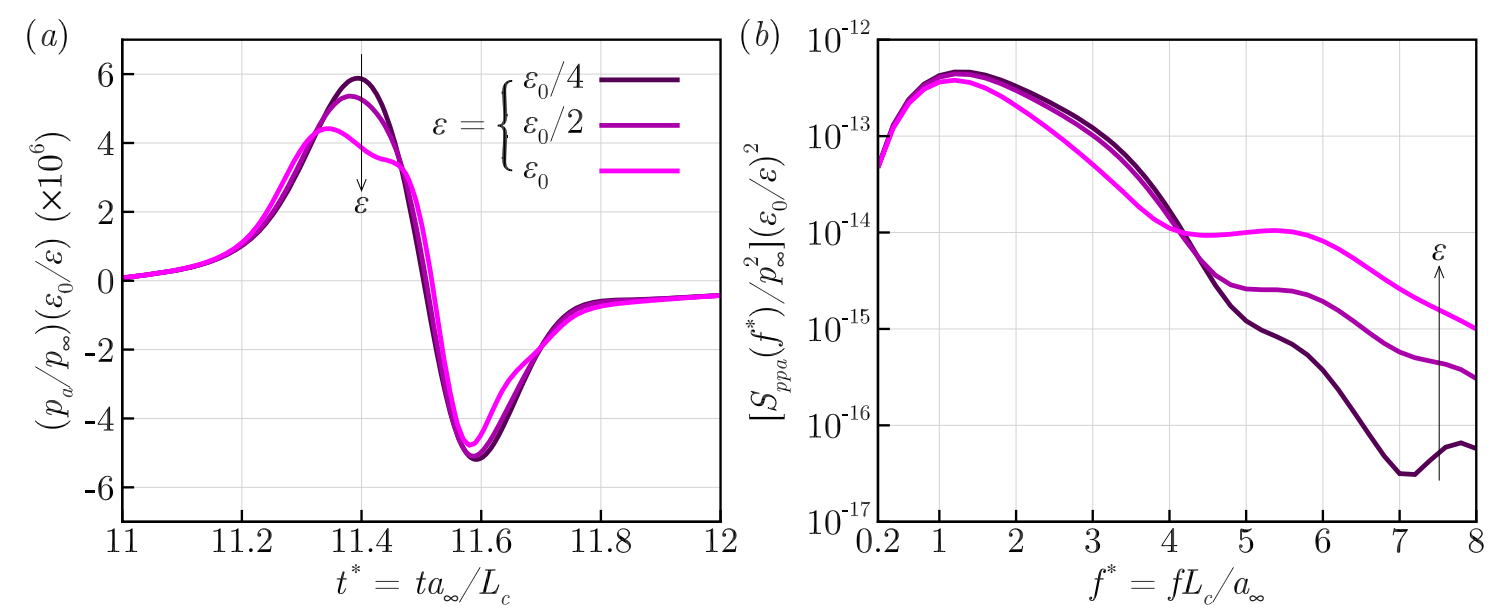

Figure 15: Far-field acoustic pressure resulted from the source at the wall shown in Fig. 14, varying with the strength of the impinging vortex: $(a)$ a close-up view of the TEVS noise signatures (see Figs. $5 b$ ) and (b) the TEVS sound power spectra, obtained at the observer position $\boldsymbol{x}_{\mathrm{\circ}} / L_{c}=(0,5)$; the acoustic pressure is re-scaled by $\epsilon_{0} / \epsilon$ for comparison purposes.

on the aerofoil surface are displayed in Fig. 13. The figure clearly exhibits the growth of the high-frequency contents and the decay of the low-frequency as the induced near-wall vortices travel downstream. This spectral change indicates a nonlinear effect. This trend is consistent on both the upper and lower surfaces although the lower surface sees a stronger growth in the high-frequency range. The opposite would have occurred if the impinging vortex had the opposite rotation. It is remarkable to observe that the major part of the vortical evolution (in terms of the spectral changes) is completed within around the first $20 \%$ of the chord, i.e. $x / L_{c} \in[-0.5,-0.3]$, followed by rather moderate/minor changes during the rest of the journey downstream to the TE. The vortical evolution that results in a significantly increased level of high-frequency perturbations is projected in the TEVS noise dominant at the high frequencies. 

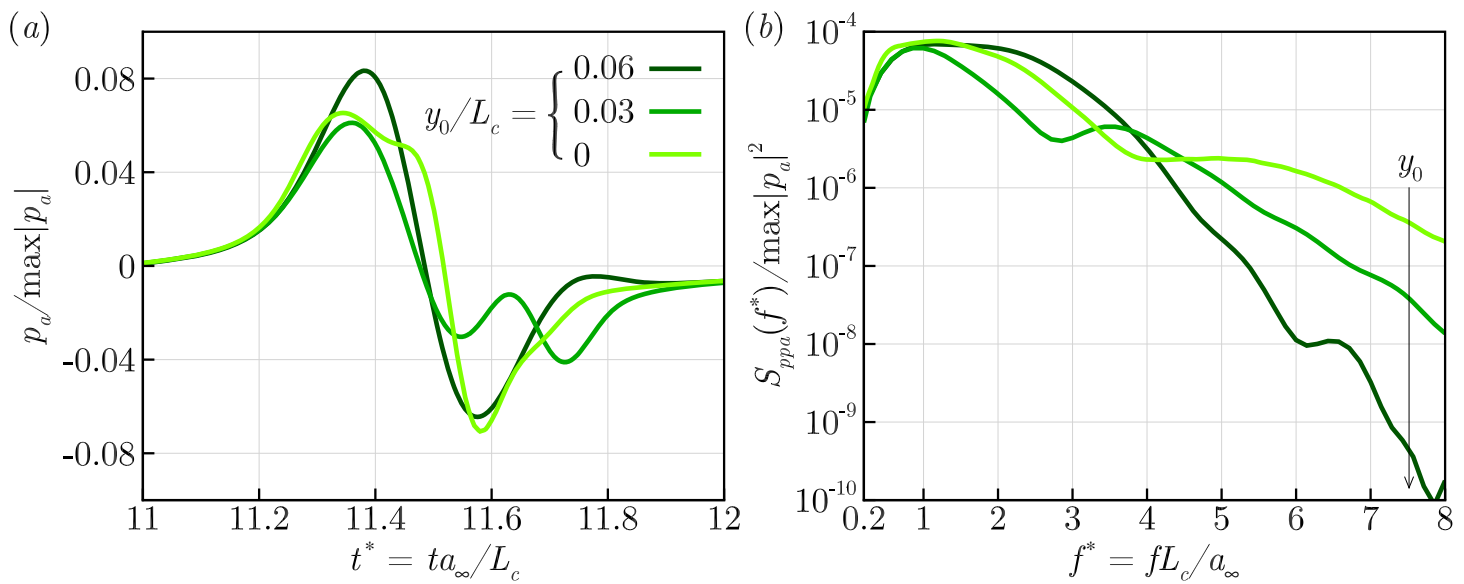

Figure 16: Far-field acoustic pressure obtained from three different cases of the vertical offset of the impinging vortex $\left(y_{0} / L_{c}=0,0.03\right.$ and 0.06 - see Fig. 1 $\left.(b)\right)$ : $(a)$ a close-up view of the TEVS noise signatures (see Figs. $5 b$ ) and $(b)$ the TEVS sound power spectra, obtained at the observer position $\boldsymbol{x}_{\mathrm{o}} / L_{c}=$ $(0,5)$; the acoustic pressure is re-scaled by the maximum amplitude of the time signal $\left(\max \left|p_{a}\left(\boldsymbol{x}_{\mathrm{o}}, t\right)\right|\right)$ for comparison purposes.

\subsection{The effect of impinging vortex strength}

Another investigation is made with regard to the strength of the impinging vortex affecting the high-frequency characteristics of the TEVS noise. Two additional simulations (Cases 2 and 3 in Table 1) are carried out where the strength of the impinging vortex is reduced to $50 \%$ and $25 \%$ of the baseline case, respectively. Fig. 14 shows the result of the additional simulations. The figure displays a clear trend that the relative amplitude of the high-frequency contents to that of the low-frequency rapidly diminishes when a weaker impinging vortex is used. This indicates that the baseline case contained a sufficiently high level of nonlinear effects to exhibit the high-frequency dominance of the TEVS noise, although the initial velocity fluctuation was just $2.5 \%$ of the free-stream. It is suggested that the nonlinear effect is attributed to the nature of the vortex dynamics that has taken place in the evolution of the induced near-wall vortices as discussed earlier. The resulting far-field acoustic pressure is also shown in Fig. 15. It is evident from the figure that the high-frequency dominance of the TEVS noise is strongly correlated with the nonlinear effect that is governed by the strength of the impinging vortex.

\subsection{The effect of vertical offset}

It is reasonable to expect that the nonlinear effect is strongest when the impinging vortex has zero vertical offset against the aerofoil surface and it becomes weaker as the vertical offset gets larger. This is because the surface-normal/vertical component of the induced velocity impinging at the LE decreases (similarly to the case of reducing the vortex strength) as the vertical offset increases. In order to confirm this, two additional simulations (Cases 4 and 5 in Table 1) are carried out where the impinging vortex has a vertical offset of $y_{0} / L_{c}=0.03$ and 0.06 , respectively. The vertical offset is defined in Eq. (6) and in Fig. 1(b). As indicated in Section 2.3, the effective radius of the impinging vortex used in this paper is $0.08 L_{c}$ and therefore the displaced vortices still make a reasonable velocity perturbation to the aerofoil. The effect of the non-zero vertical offset is revealed in Fig. 16. It is clear from the figure that the high-frequency contents of the TEVS noise diminish rapidly with increasing the vertical offset, similarly to the earlier case of reducing the vortex strength (Fig. 15). 
With regard to the nonlinear effect, we draw attention to the similar findings made by Grace 29. She suggested that linear assumptions were invalid when the impinging vortex strength was high or the vertical offset was small. She proposed a rule-of-thumb criterion for the nonlinear regime - "vortices with strength $n \%$ of the freestream passing less than $n \%$ of a halfchord away from the blade". Applying this criterion to the current case $\left(\max |v| / u_{\infty}=0.025\right)$ would predict $y_{0} / L_{c}=0.0125$ above which the radiated noise should begin to scale linearly. However, the current result still exhibits significant changes to the spectra shape at larger values of the vertical offset. This is attributed to the distributed vortex model with a radius of approximately $0.08 L_{c}$ (as opposed to zero for a point vortex).

It appears that increasing the vertical offset with the vortex strength fixed produces a similar effect of decreasing the vortex strength at zero offset, at least with respect to the growth/decay of the TEVS noise in the high-frequency range. Based on this finding, the vertical offset test is not repeated in the following viscous flow studies which are computationally demanding.

\section{The effect of viscosity on TEVS noise}

This section presents viscous solutions of the aerofoil-vortex interaction in order to study how the viscosity affects/changes the high-frequency dominance of the TEVS noise identified above from the inviscid solutions. As indicated in Table 1 (Cases 6 to 9), 3-D Navier-Stokes simulations are carried out for a Reynolds number of $R e_{\infty}=\rho_{\infty} u_{\infty} L_{c} / \mu_{\infty}=$ $4 \times 10^{5}$ which is typically of the order of a small/medium low-speed wind-tunnel experiment. At this Reynolds number, the boundary layers on the flat plate are likely to remain laminar [58] during and after the vortex's interactions with the aerofoil (at least within the time frame of the current simulations). This scenario eliminates the possibility of generating "self-noise" (due to turbulent boundary layers scattered at the TE) to interfere with the genuine TEVS noise that is the main subject of this study. On the other hand, there is a possible interference from "wake noise" that is a quadrupole type of noise. The wake noise may appear noticeable in the high-frequency range. After evaluating the contribution of the wake noise in the current acoustic data, this section provides detailed information about the TEVS noise at the high frequencies under the viscous flow conditions.

\subsection{Base flow and wake noise}

Since the uniform base flow used in the inviscid cases is no longer applicable in the viscous cases, a separate precursor simulation is required to generate the viscous base flow (Case 6). From the precursor simulation carried out without the impinging vortex, a quasisteady-state solution is obtained. The resulting solution gives fully developed laminar boundary layers on both sides of the flat-plate aerofoil and the subsequent laminar wake downstream of the TE which adds a weak unsteady fluctuation to the base flow. Fig. 17 shows the result of the precursor simulation for the base flow which accurately reproduces the Blasius solution except very near the TE where the flat plate ceases to exist. In the meantime, the level of wake noise generated from the base flow is quantified in Fig. 18 compared with that of the total noise (LEVS+TEVS+ABS and the wake noise) from Case 7. It is exhibited in the figure that the wake noise may become significant in the high frequency range $\left(f^{*}>7\right.$ in Fig. $\left.18 b\right)$. The relative impact of the wake noise depends on the Reynolds and Mach numbers of the base flow. It is possible to exclude (albeit not perfectly) the wake noise contribution from the noise spectra by using a Ffowcs WilliamsHawkings (FW-H) formulation [59] based on the dipole sources integrated over the aerofoil 

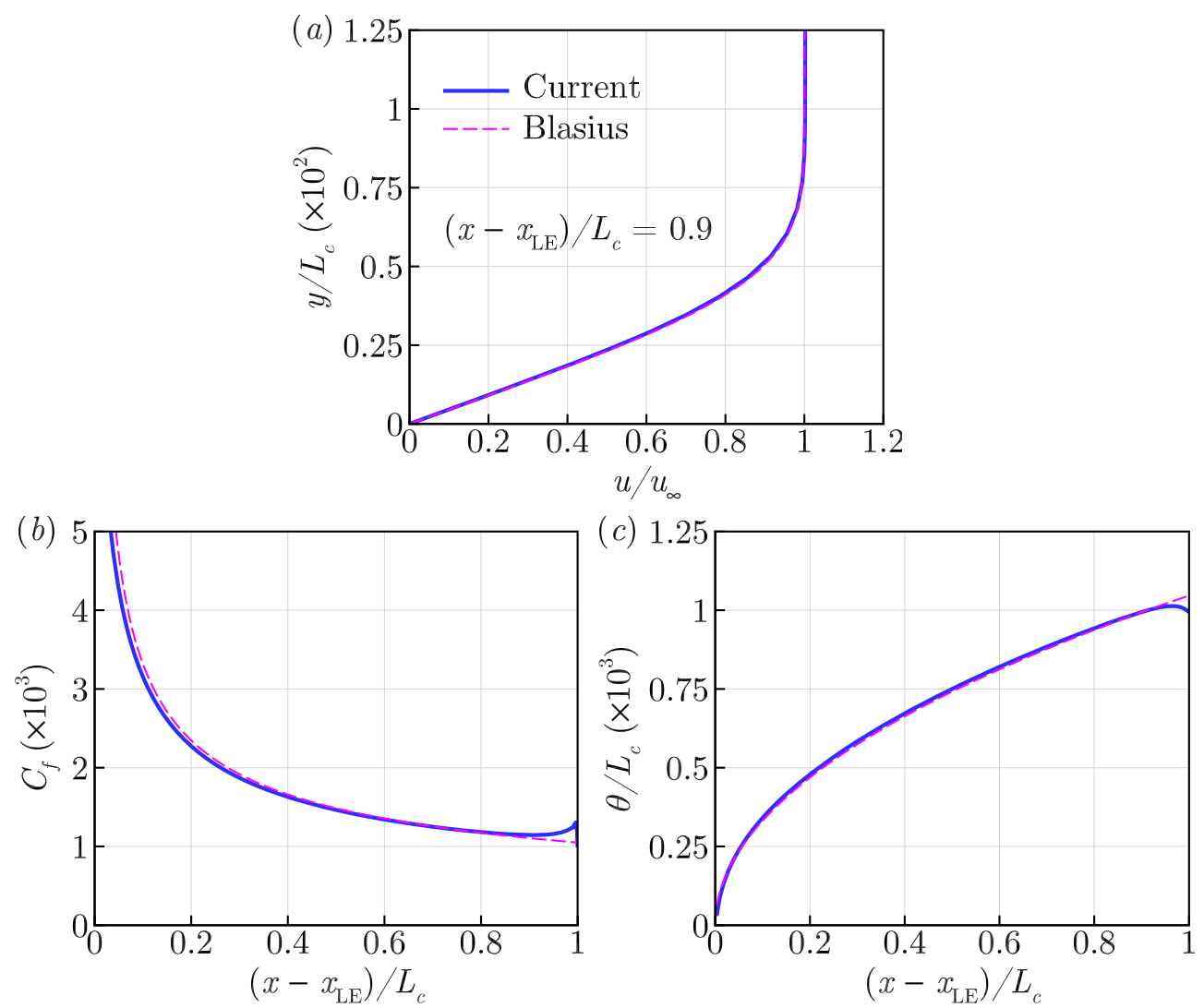

Figure 17: Laminar boundary layer generated as the base flow (Case 6) of the current viscous simulations: (a) the streamwise velocity profile probed at $90 \%$ of the chord, $(b)$ the distribution of skin friction coefficient and $(c)$ the momentum thickness along the chord, compared against the Blasius solution.
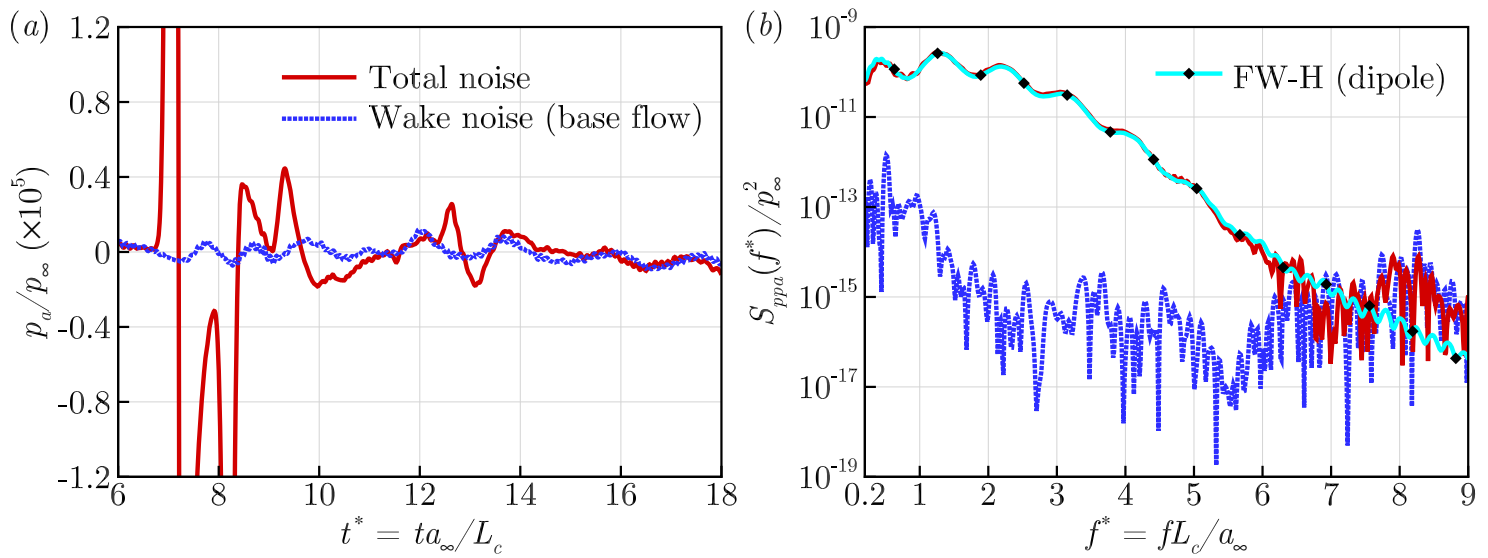

Figure 18: Wake noise generated from the base flow (Case 6) compared to the total noise (LEVS+TEVS+ABS and the wake noise from Case 7) - both cases with a finite aerofoil: $(a)$ the time signals obtained at $\boldsymbol{x}_{\mathrm{o}} / L_{c}=(0,5,0)$ and $(b)$ the corresponding sound power spectra; the Ffowcs WilliamsHawkings $(\mathrm{FW}-\mathrm{H})$ spectrum in $b$ is calculated based on the dipole sources integrated over the aerofoil surface 59 . 


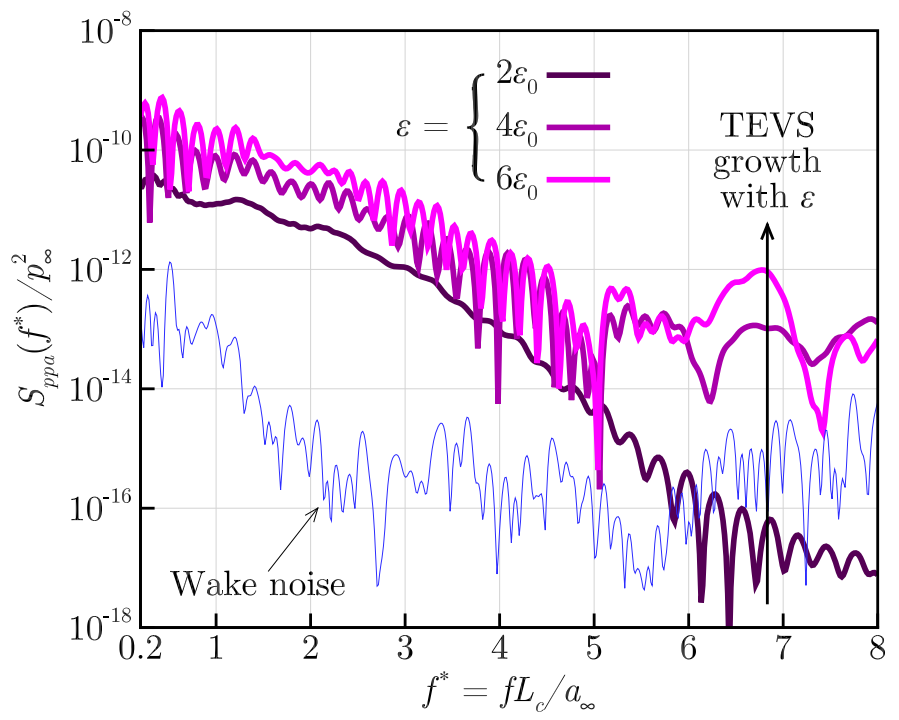

Figure 19: High-frequency TEVS noise growing under the viscous flow condition with the strength of the impinging vortex increased; the spectra are calculated based on the secondary sources (TEVS+ABS) obtained from Eq. 14.

surface. This approach still contains a minor contribution of the wake noise scattered by the aerofoil. The result of the FW-H calculation is plotted in Fig. $18(b)$, which shows an almost perfect match with the total noise spectrum up to $f^{*} \approx 6$ followed by a consistent decay in the high-frequency range as expected without the wake noise contribution. Herein, all noise spectra are calculated by using the dipole $\mathrm{FW}-\mathrm{H}$ formulation in order to avoid interference by the wake noise.

\subsection{Viscous dissipation versus impinging vortex strength}

It is worth noting that Case 7 demonstrated in Fig. 18 is based on a vortex which is twice as strong as the one used in the inviscid case $\left(\epsilon=2 \epsilon_{0}\right)$. The result, however, does not show a sign of TEVS appearing at the high frequencies $\left(f^{*}>5\right)$ as it was in the inviscid case despite the increased strength of the impinging vortex. It is worth noting that the increased vortex strength is still much weaker than what is normally expected in rotorcraft BVI noise $\left(\epsilon=12 \epsilon_{0}\right.$ to $\left.22 \epsilon_{0}\right)$ resulting in an induced velocity of $30 \%$ to $55 \%$ of the freestream velocity as reported in the literature (see Section 1). Anyhow, the result suggests that the vortical disturbances have been dissipated by the viscosity before they reach the TE. In Section 4.1, it has been learnt that induced near-wall vortices are responsible for the high-frequency dominance of TEVS noise (Figs. 12 and 14). The height of the induced near-wall vortices was around one or two per cent of the aerofoil chord as displayed in Fig. 12 and it is in the same order of the boundary layer thickness near the TE as shown in Fig. 17(a). Therefore, in the current simulation set-up, it is reasonable to guess that the most part of the near-wall vortices would have travelled through the boundary layer and undergone stretching-dissipation processes.

When the vortex strength is increased for the induced near-wall vortices to survive the dissipation, the TEVS noise appears again. Fig. 19 shows a sudden growth of TEVS noise at high frequencies $\left(f^{*}>5\right)$ when the vortex strength is increased. The wake noise spectrum is also plotted in the same figure to clarify the relative significance of the TEVS noise at the high frequencies. With the increased vortex strengths $\epsilon=4 \epsilon_{0}$ and $6 \epsilon_{0}$, the induced velocity perturbation (prior to the interaction) reaches up to $10 \%$ and $15 \%$, respectively, of the free-stream velocity. Although this level of velocity perturbation 
(a)

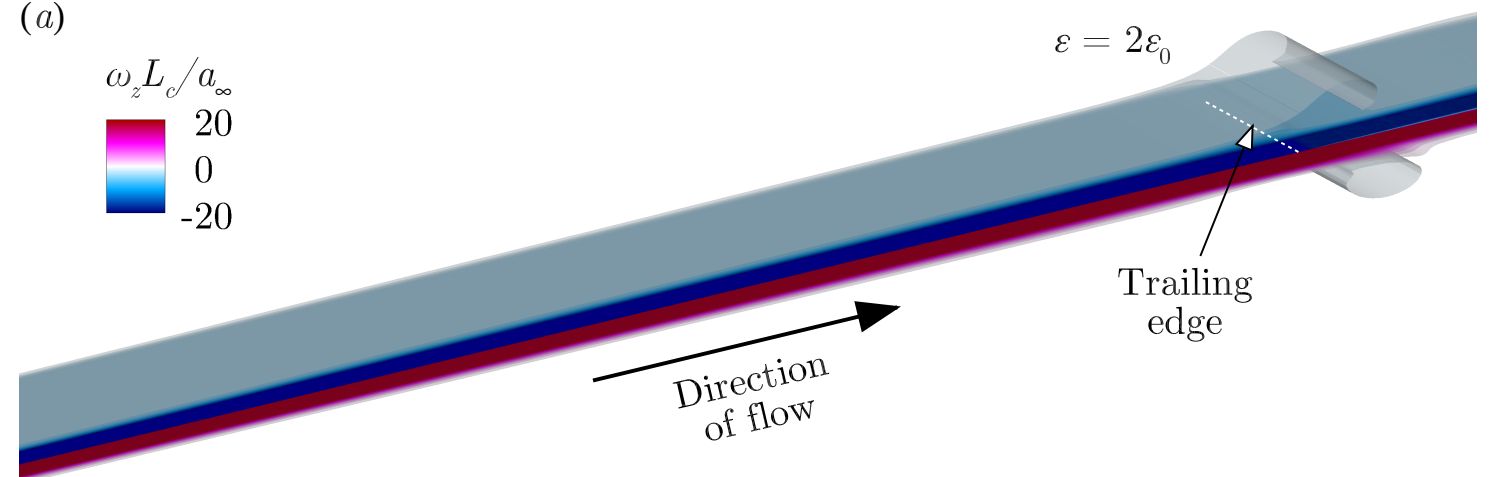

(b)

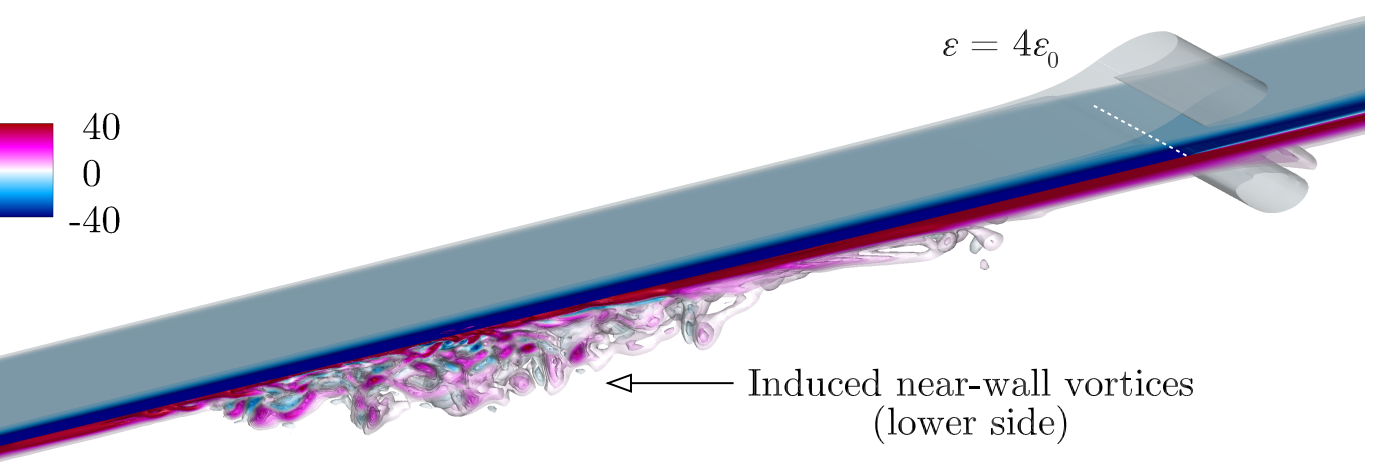

$(c)$

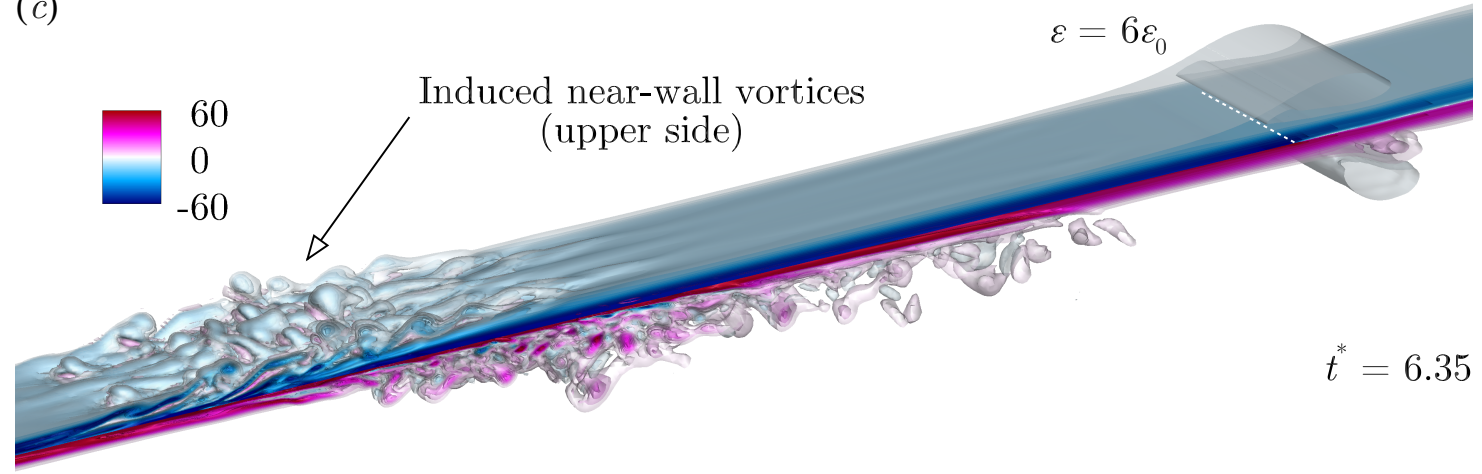

Figure 20: Instantaneous spanwise vorticity iso-surfaces in the vicinity of the TE taken when the initial vortex passes through the TE, for three different vortex strengths: $(a) \epsilon=2 \epsilon_{0},(b) 4 \epsilon_{0}$ and $(c) 6 \epsilon_{0}$.

is still weaker than those studied in rotorcraft blade-vortex interactions (up to $55 \%$ as mentioned in Section 1), the zero vertical offset in the current cases means more efficient production of the nonlinear effects. With the vortex strength increased, it is worth noting that the level of TEVS noise is up to 20 or $30 \mathrm{~dB}$ higher than that seen in the inviscid case (with $\epsilon=\epsilon_{0}$ ) if Figs. 6 and 19 are compared in the high-frequency range $\left(f^{*}>5\right)$. In the meantime, we have re-examined the boundary layers when all interactions came to an end and confirmed that the boundary layers remained laminar (the same as the initial Blasius solution shown in Fig. 17).

\subsection{Induced near-wall vortices in viscous flow}

In order to understand the mechanisms by which the increased vortex strength leads to the resurgence of high-frequency TEVS noise in the viscous flow, the vorticity fields near the TE are plotted in Fig. 20, comparing the three different cases: $\epsilon=2 \epsilon_{0}, 4 \epsilon_{0}$ and $6 \epsilon_{0}$. First, it is apparent that the weaker vortex case $\left(\epsilon=2 \epsilon_{0}\right)$ shows no sign of induced 
near-wall vortices surviving at the TE on either sides of the aerofoil, hence insignificant TEVS noise. In the meantime, as the initial vortex strength is increased $\left(\epsilon=4 \epsilon_{0}\right.$ and $\left.6 \epsilon_{0}\right)$, some of the induced near-wall vortices manage to survive (or rather amplify themselves) and reach the TE. Also, it is evident that there is a strong three-dimensionality in the vortical structures created which has not been present in the inviscid study. The smaller three-dimensional structures naturally give rise to the level of disturbances at the high frequencies leading to the resurgence of the high-frequency TEVS noise. However, this event has a short finite lifespan which is clearly different from the self-noise mechanism. Secondly, it is interesting to observe that the induced near-wall vortices may appear only on one side of the aerofoil (rather than on both), which may depend on the initial vortex strength, direction of rotation and the Reynolds number. The current vortex which rotates clockwise generates a downwash first and an upwash later at the LE of the aerofoil. The opposite rotation would have resulted in a vertically reversed solution. Thirdly, in Fig. 20(c), it is noticeable that there is a significant difference in the streamwise locations between the upper and lower vortices. Also, they are travelling slower than the initial vortex, which has not been the case in the earlier inviscid flow scenario. These distinctive features are partially contributed by the wall image/mirror effect which is more significant due to the stronger vortex used, and also by the slower near-wall convection speed (within the boundary layer) compared to the free-stream speed.

Fig. 21 provides some more details of the strong vortex case $\left(\epsilon=6 \epsilon_{0}\right)$ seen in Fig. 20(c). The mid-span cross-sectional snapshots reveal that the near-wall vortices begin to appear (bulging out of the boundary layer) and grow on the lower side of the aerofoil first. Meanwhile, the upper side experiences a delayed growth of the near-wall vortices, emerging from about the mid-chord. The vortices seem to keep growing while travelling downstream until they reach the TE and get scattered there. The growth of the near-wall vortices under the viscous dissipation is a nonlinear process that did not take place in the weaker vortex case $\left(\epsilon=2 \epsilon_{0}\right)$. It is envisaged that there is a local convective instability threshold above which the nonlinear effect takes over the dissipation effect [60], which could be investigated in detail as a separate study. However, the current numerical simulations did not exhibit a sign of absolute instability triggering a turbulent boundary layer due to the near-wall vortices. As mentioned earlier, the boundary layers remained laminar after the vortices finished their interactions with the TE and left the computational domain. In the absence of self-noise sources, the viscous TEVS noise generation mechanism - induced near-wall vortices containing high-frequency contents - is consistent with the inviscid mechanism identified earlier in this study. Fig. 22 visualises the acoustic waves generated by the scattering of the induced near-wall vortices at the TE (in comparison with those by the initial vortex of $\epsilon=6 \epsilon_{0}$ ). The outcome of the strong TEVS noise signature is displayed in the sound power spectrum provided in Fig. 19.

\subsection{On the tonal noise component}

The dominance of TEVS appears particularly strong around $f_{p}^{*}=6.8$ in Fig. 19 . There is a possibility that it is a spectral peak (rather than a broadband hump) due to a tonal noise component. In order to verify this, the following procedure is taken. First, we calculate a Fourier transform of the TEVS solution at every single grid point. Second, we extract a narrow band of the high-frequency contents within $f_{p}^{*}-\Delta f^{*}<f^{*}<$ $f_{p}^{*}+\Delta f^{*}$ (centred at $\left.f_{p}^{*}=6.8\right)$ by multiplying a frequency filter (weighting function) to the Fourier transform. Finally, we calculate an inverse Fourier transform of it, i.e. a filtered TEVS solution as a function of time and space, which contains the narrow-band 

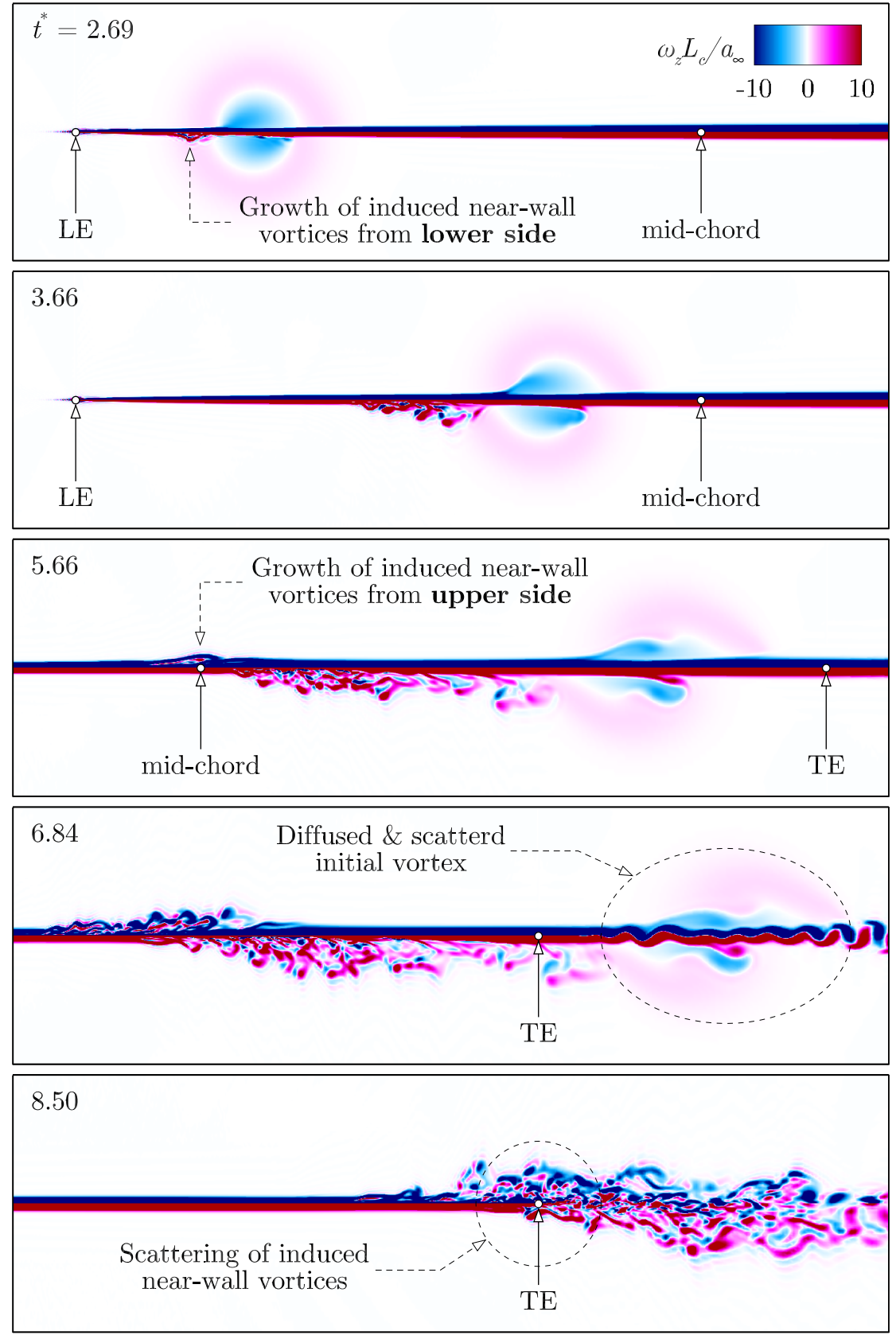

Figure 21: Instantaneous snapshots of spanwise vorticity contours $\left(\omega_{z} L_{c} / a_{\infty}\right)$ taken at the mid-span cross-section, showing the growth and evolution of near-wall vortices induced by the initial vortex passing through the boundary layer; the case with $\epsilon=6 \epsilon_{0}$ (see Fig. 20 c) is plotted here. 
(a)

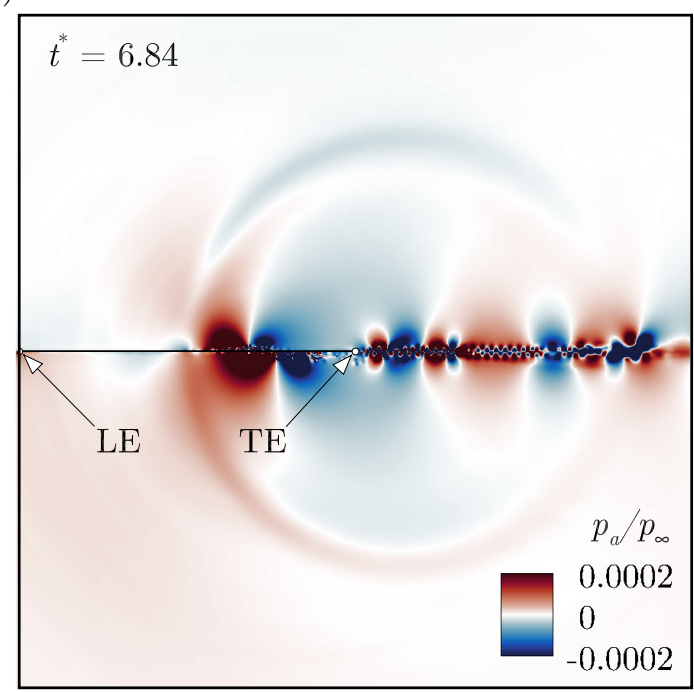

$(b)$

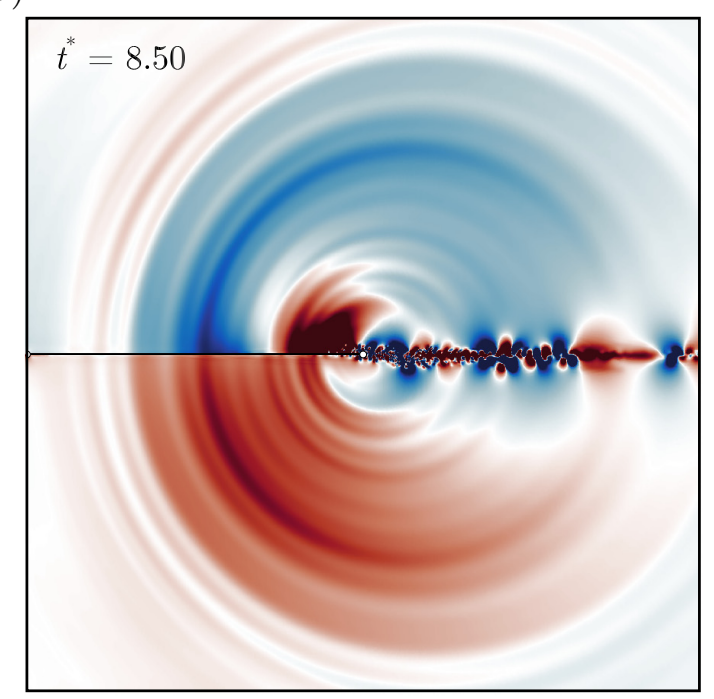

Figure 22: Instantaneous snapshots of acoustic pressure contours $\left(p_{a} / p_{\infty}\right)$ taken at the mid-span crosssection, at two different points in time: $(a) t^{*}=6.84$ and $(b)$ 8.50, which correspond to the last two plots of Fig. 21. showing the sound waves generated by the scattering of the initial vortex at the TE (left) and that of the near-wall vortices (right), respectively.

(a)

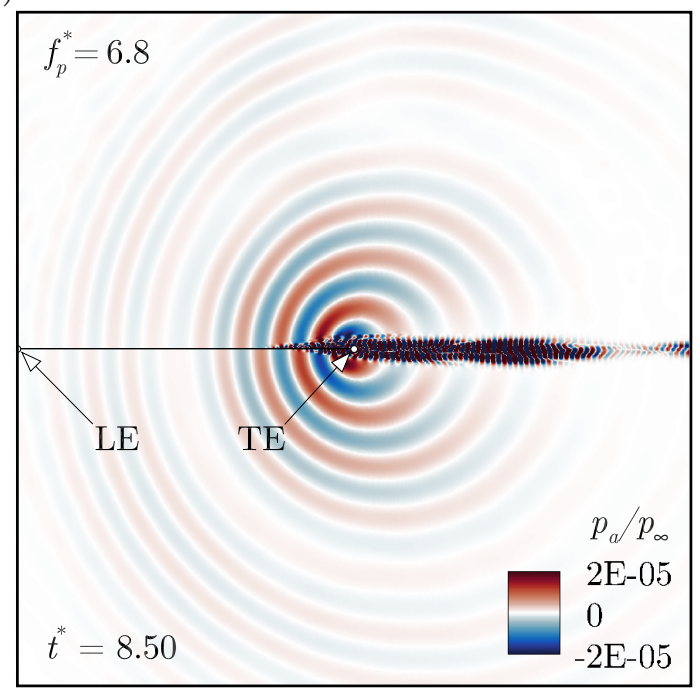

(b)

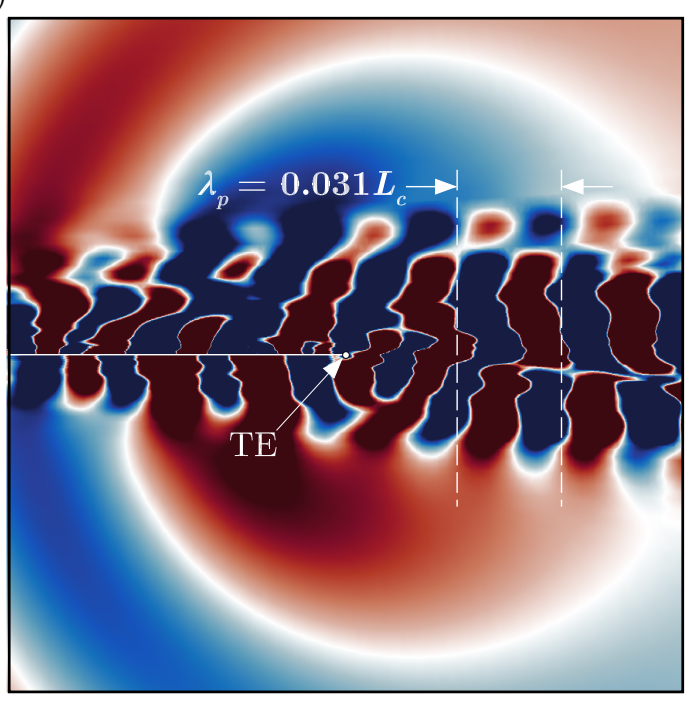

Figure 23: A frequency-filtered TEVS solution extracted from the original one (Fig. 222) to visualise the tonal noise feature around $f_{p}^{*}=6.8$ indicated in the sound power spectra (Fig. 19): $(a)$ an instantaneous snapshot taken at $t^{*}=8.50$ and $(b)$ an additional close-up view near the TE; only a narrow band of frequency contents $\left(f_{p}^{*}-0.5 \leq f^{*} \leq f_{p}^{*}+0.5\right)$ are contained in this figure; the case with $\epsilon=6 \epsilon_{0}$ is plotted here. 


\begin{tabular}{cccccc}
\hline & $\left(x_{0}-x_{\mathrm{LE}}\right) / L_{c}$ & $\delta_{1} / L_{c}$ & $R e_{\delta_{1}}$ & $\max \left(\alpha_{r} \delta_{1}\right)$ & $\lambda_{\min } / L_{c}$ \\
\hline Upper side & 0.392 & 0.00174 & 696 & 0.333 & 0.0328 \\
Lower side & 0.214 & 0.00130 & 520 & 0.301 & 0.0271 \\
\hline
\end{tabular}

Table 2: A prediction of the shortest wavelength of the convective instability modes in the induced nearwall vortices based on an Orr-Sommerfeld solution [41, 61; here, $x_{0}, \delta_{1}$ and $\alpha_{r} \delta_{1}$ denote the critical point (where the near-wall vortices first appear), the boundary-layer displacement thickness at the critical point and a dimensionless wavenumber of the instability modes (obtained from the literature), respectively; the wavelength of the instability mode is $\lambda=2 \pi \delta_{1} /\left(\alpha_{r} \delta_{1}\right)$.

frequency contents only:

$$
\begin{gathered}
\widetilde{p_{a}}(\boldsymbol{x}, t)=\mathcal{F}^{-1}\left\{W(f) \mathcal{F}\left[p_{a}(\boldsymbol{x}, t)\right]\right\}, \\
W(f)=\sum_{m=0}^{1} \exp \left[-(\ln 10)\left(f+(-1)^{m} f_{p}\right)^{2} / \Delta f^{2}\right],
\end{gathered}
$$

where $\mathcal{F}$ and $\mathcal{F}^{-1}$ represent the Fourier and inverse Fourier transforms, respectively; and, $W(f)$ is the narrow-band frequency filter with a bandwidth set to $\Delta f^{*}=1$. An instantaneous snapshot of the filtered TEVS solution is shown in Fig. 23. A clear signature of a tonal (periodic) sound generation is visualised in this figure. When viewed more closely in the vicinity of the TE, identified is a periodic flow structure (consisting of the near-wall vortices) with a wavelength of $\lambda_{p}=0.031 L_{c}$ passing through the TE. The convection speed of this flow structure is $u_{p}=f_{p} \lambda_{p}=0.211 a_{\infty}$. This is $88 \%$ of the free-stream speed, which indicates a fairly fast-moving secondary structure.

We suggest that the origin of this particular wavelength component in the near-wall vortices can be identified from an Orr-Sommerfeld solution for a flat-plate boundary layer [41, 61]. We assume that there are convective instability modes triggered at the critical locations where the near-wall vortices started to appear (as seen in Fig. 21). The wavelengths of the instability modes are predicted from the Orr-Sommerfeld solution based on the displacement thickness $\left(\delta_{1}\right.$ and $R e_{\delta_{1}}$ ) of the undisturbed boundary layer at those critical locations. Amongst a range of wavelengths that are predicted to have contributed to the instability, we pursue the shortest wavelength commensurate with the small size of the perturbed flow structure (of the order of $\delta \approx 3 \delta_{1}$ ) when they start to appear. The result of the prediction is summarised in Table 2. It is found in the table that the predicted wavelengths from both the upper and lower sides of the aerofoil are fairly close to the wavelength of the dominant TEVS source $\left(\lambda_{p} / L_{c}=0.031\right)$ identified in Fig. 23.

\section{Concluding remarks}

The present high-resolution numerical simulations provided us with detailed information on the trailing-edge noise generation in the event of interaction between a flat-plate aerofoil and an impinging vortex. Both inviscid and viscous flow conditions were investigated, from which comprehensive understandings were obtained. One of the main conclusions of this study is that the production of TEVS noise (as a secondary source of the aerofoil-vortex interaction noise) is a nonlinear process, which was somehow predicted in the previous analytical studies albeit based on different nonlinear aspects. In the current study, although the LEVS was still the primary source of the aerofoil-vortex interaction noise (dictating the low-to-mid frequencies), it was found that the TEVS noise could exceed the primary noise in the high-frequency range $\left(f^{*}>5\right)$. The high-frequency dominance of the TEVS noise took place almost omni-directionally except within a narrow window of downstream angles. This finding may have significant implications for studies 
of aerofoil-turbulence interaction noise since the TE scattering of the impinging turbulence has historically been neglected. Unlike the LEVS noise, the TEVS noise showed significant nonlinear changes in its power spectrum in the high-frequency range when the strength and/or vertical offset of the impinging vortex were changed. We identified that this event was due to the dynamics/evolution of the near-wall vortices induced during the collision/interaction of the impinging vortex with the aerofoil. The frequency contents of the near-wall vortices changed substantially as they were travelling downstream from the LE to TE. The high-frequency noise spectrum at the far field almost perfectly correlated with the near-wall vorticity spectrum probed at the TE.

The high-frequency dominance of the TEVS noise also appeared in the viscous flow condition when the near-wall vortices survived the viscous dissipation. This means that the strength of the impinging vortex needed to be higher than that of the inviscid flow to be able to trigger the nonlinear effect. At the Reynolds number selected in this study $\left(R e_{\infty}=4 \times 10^{5}\right)$, the impinging vortex with a strength of $\epsilon=4 \epsilon_{0}$ produced the highfrequency dominance of TEVS noise that was significantly more pronounced (about 20 $\mathrm{dB}$ higher) than that of the inviscid case $\left(\epsilon=\epsilon_{0}\right)$. However, the other case with $\epsilon=2 \epsilon_{0}$ produced no sign of TEVS noise at all frequencies. This step change between $\epsilon=2 \epsilon_{0}$ and $4 \epsilon_{0}$ in the viscous flow condition was attributed to the nonlinear growth of the near-wall vortices associated with the local convective instability of the boundary layer. The OrrSommerfeld solution correctly predicted the instability wavelength that was responsible for the peak frequency of the TEVS noise at $f^{*}=6.8$ in the current study. It was interesting to observe that the nonlinear growth of the near-wall vortices could take place only on one side of the aerofoil, which should depend on the Reynolds number, the strength and direction of the vortex rotation. The viscous effect created a highly 3 -D structure of the near-wall vortices (when triggered) resulting in a broader high-frequency response at the TE, compared to the 2-D inviscid cases.

The new findings from the inviscid cases may have strong relevance to theoretical or semi-analytical approaches if they are to be further improved or modified in order to accurately describe the TEVS noise contribution. On the other hand, the viscous results naturally have more relevance to real-life applications. One of obvious examples is the BVI (blade-vortex interaction) noise in rotorcraft where the vortex strength reaches up to $22 \epsilon_{0}\left(\left|v^{\prime}\right|_{\max }=0.55 u_{\infty}\right)$ and the impulsive signatures of TEVS noise which dominate over other sources (i.e. self-noise) have been reported in the literature as outlined in Section 1. The present results support them. On the other hand, it is unclear so far how the TEVS noise will compare with the self-noise when the vortex strength is sufficiently low so that its induced velocity is comparable to the boundary layer's turbulence intensity. This will be a challenging study since there are additional nonlinear interactions expected to take place between the vortex and the turbulent boundary layer (as well as the transition beforehand). The current results may become a reference point for someone who undertakes such complex investigations. In addition, the inviscid and viscous solutions produced in this work will serve as useful benchmark data and baseline test cases for future studies based on more realistic aerofoil geometries and/or flow conditions.

\section{Acknowledgement}

We would like to thank EPSRC (Engineering and Physical Sciences Research Council) for the support of Doctoral Training Centre in Complex Systems Simulations (EP/G03690X/1), and for the computational time made available on the UK supercomputing facility ARCHER via the UK Turbulence Consortium (EP/R029326/1). We also acknowledge the use of the IRIDIS High Performance Computing Facility and associated support services 


\begin{tabular}{cccccccr}
\hline$R e_{\infty}$ & Grid resolution & $N_{\xi}$ & $N_{\eta}$ & $N_{\zeta}$ & $\Delta x_{\min } / L_{c}$ & $\Delta y_{\min } / L_{c}$ & $\mathrm{CPW}$ \\
\hline inviscid & coarse & 1280 & 768 & - & 0.0024 & 0.0024 & 3.93 \\
inviscid & medium & 1600 & 960 & - & 0.0020 & 0.0020 & 5.71 \\
inviscid & fine (current) & 1760 & 1056 & - & 0.0018 & 0.0018 & 6.74 \\
$4 \times 10^{5}$ & coarse & 1600 & 1120 & 30 & 0.002 & 0.00035 & 7.13 \\
$4 \times 10^{5}$ & medium & 1920 & 1232 & 34 & 0.0018 & 0.000315 & 8.52 \\
$4 \times 10^{5}$ & fine (current) & 2240 & 1344 & 38 & 0.0016 & 0.00028 & 10.02 \\
\hline
\end{tabular}

Table A.3: Grid convergence test matrix for the current inviscid and viscous simulations (Cases 1 and 8 in Table 1); $N_{\xi}, N_{\eta}$ and $N_{\zeta}$ denote the number of grid cells used in the streamwise, vertical and spanwise directions, respectively; $\Delta x_{\min }$ and $\Delta y_{\min }$ represent the grid resolution on the aerofoil surface; CPW (cells per wavelength) indicates the grid resolution at the far field for sound waves approaching the observer location $\boldsymbol{x}_{o} / L_{c}=(0,5,0)$ at the frequency of $f^{*}=8$ that is the highest valid frequency considered in this paper; the spanwise resolution of the 3 -D cases (viscous simulations) is kept unchanged; the "fine" resolutions are used throughout the paper.
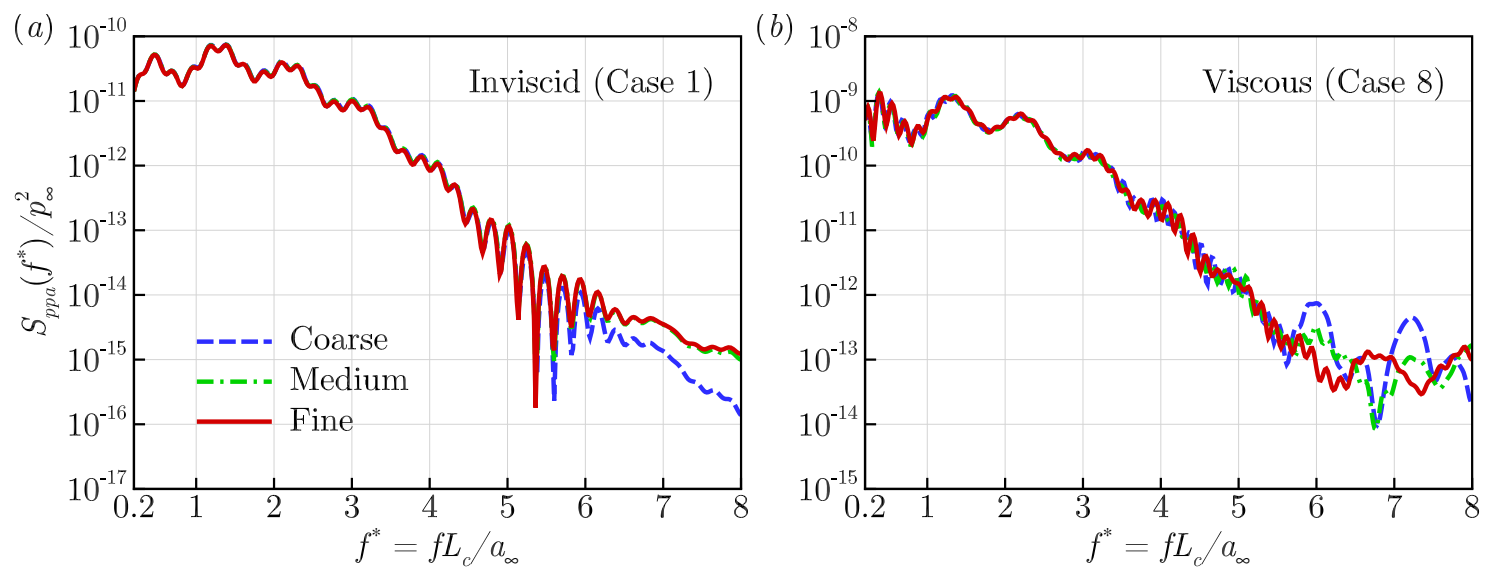

Figure A.24: Sound power spectra obtained at the observer location $\boldsymbol{x}_{o} / L_{c}=(0,5,0)$ based on three different levels of grid resolution as described in Table A.3 from the $(a)$ inviscid and $(b)$ viscous simulations (Cases 1 and 8 in Table 1).

at the University of Southampton in the completion of this work. All data supporting this study are openly available from the University of Southampton repository at http://dx.doi.org/10.5258/SOTON/XXXXXX.

\section{Appendix A. Validation of the grid resolution used}

The grid quality of the current simulations is examined here. Three different (coarse, medium and fine) levels of the grid resolution are tested for both the inviscid and viscous simulations (Cases 1 and 8 in Table 1). The fine resolution is used as standard in this paper. The details of the grids used in this test are provided in Table A.3. The result of the test simulations is shown in Fig. A.24 in terms of the radiated sound power spectra. It is displayed that all three resolution levels produce almost identical spectra for frequencies up to about $f^{*} \approx 5.5$. However, the coarse grid exhibits a noticeable deviation from the other two in the high frequency range where the TEVS noise is significant. The agreement between the medium and fine grids is consistent throughout the entire frequency range (up to $f^{*}=8$ ) considered in this paper, although the viscous case displays minor/moderate oscillations $\left(f^{*}>5.5\right)$ that are not perfectly matched. The high-frequency spectral oscillations are related to interferences from the wake noise. Apart from the oscillatory mismatches, the level of the high-frequency TEVS noise is well captured with both the medium and fine grids. 
[1] R. K. Amiet, Acoustic radiation from an airfoil in a turbulent stream, J. Sound Vib. 41 (1975) 407-420.

[2] M. Roger, S. Moreau, Extensions and limitations of analytical airfoil broadband noise models, Int. J. Aeroacoust. 9 (2010) 273-305.

[3] K. Kucukcoskun, J. Christophe, C. Schram, M. Tournour, Broadband scattering of the turbulence-interaction noise of a stationary airfoil: experimental validation of a semi-analytical model, Int. J. Aeroacoust. 12 (2013) 83-102.

[4] L. Santana, C. Schram, W. Desmet, Low-frequency extension of Amiet's theory for compact noise predictions, J. Sound Vib. 372 (2016) 342-356.

[5] M. E. Goldstein, Unsteady vortical and entropic distortions of potential flows around arbitary obstacles, J. Fluid Mech. 89 (1978) 433-468.

[6] M. R. Myers, E. J. Kerschen, Influence of incidence angle on sound generation by airfoils interacting with high-frequency gusts, J. Fluid Mech. 292 (1995) 271-304.

[7] M. R. Myers, E. J. Kerschen, Influence of camber on sound generation by airfoils interacting with high-frequency gusts, J. Fluid Mech. 353 (1997) 221-259.

[8] I. Evers, N. Peake, Noise generation by high-frequency gusts interacting with an airfoil in transonic flow, J. Fluid Mech. 411 (2000) 91-130.

[9] L. J. Ayton, N. Peake, On high-frequency sound generated by gust-aerofoil interaction in shear flow, J. Fluid Mech. 766 (2015) 297-325.

[10] R. W. Paterson, R. K. Amiet, Acoustic radiation and surface pressure characteristics of an airfoil due to incident turbulence, NASA CR-2733 (1976).

[11] S. Moreau, M. Roger, V. Jurdic, Effect of angle of attack and airfoil shape on turbulence-interaction noise, in: 11th AIAA/CEAS Aeroacoustics Conference. AIAA Paper 2005-2973, 2005.

[12] W. J. Devenport, J. K. Staubs, S. A. L. Glegg, Sound radiation from real airfoils in turbulence, J. Sound Vib. 329 (2010) 3470-3483.

[13] H. M. Atassi, S. Subramaniam, J. R. Scott, Acoustic radiation from lifting airfoils in compressible subsonic flow, in: 13th Aeroacoustics Conference. AIAA Paper 90-3911, 1990 .

[14] D. P. Lockard, P. J. Morris, Radiated noise from airfoils in realistic mean flows, AIAA J. 36 (1998) 907-914.

[15] J. Christophe, J. Anthoine, P. Rambaud, C. Schram, F. Mathey, S. Moreau, Prediction of incoming turbulent noise using a combined numerical/semi-empirical method and experimental validation, in: 13th AIAA/CEAS Aeroacoustics Conference. AIAA Paper 2007-3468, 2007.

[16] J. Christophe, J. Anthoine, P. Rambaud, S. Moreau, Numerical issues in the application of an Amiet model for spanwise-varying incoming turbulence, in: 14th AIAA/CEAS Aeroacoustics Conference. AIAA Paper 2008-2865, 2008. 
[17] H. Deniau, G. Dufour, J-F. Boussuge, C. Polacsek, S. Moreau, Affordable compressible LES of airfoil-turbulence interaction in a free jet, in: 17th AIAA/CEAS Aeroacoustics Conference. AIAA Paper 2011-2707, 2011.

[18] J. Gill, X. Zhang, P. Joseph, Single velocity-component modeling of leading edge turbulence interaction noise, J. Acoust. Soc. Am. 137 (2015) 3209-3220.

[19] J. W. Kim, S. Haeri, P. Joseph, On the reduction of aerofoil-turbulence interaction noise associated with wavy leading edges, J. Fluid Mech. 792 (2016) 526-552.

[20] J. M. Turner, J. W. Kim, Aeroacoustic source mechanisms of a wavy leading edge undergoing vortical disturbances, J. Fluid Mech. 811 (2017) 582-611.

[21] J. M. Turner, J. W. Kim, On the universal trends in the noise reduction due to wavy leading edges in aerofoil-vortex interaction, J. Fluid Mech. 871 (2019) 186-211.

[22] S. W. Rienstra, Sound diffraction at a trailing edge, J. Fluid Mech. 108 (1981) 443-460.

[23] L. J. Ayton, N. Peake, On high-frequency noise scattering by aerofoils in flow, J. Fluid Mech. 734 (2013) 144-182.

[24] M. Roger, S. Moreau, K. Kucukcoskun, On sound scattering by rigid edges and wedges in a flow, with applications to high-lift device aeroacoustics, J. Sound Vib. 362 (2016) 252-275.

[25] D. G. Crighton, Radiation from a vortex filament motion near a half plane, J. Fluid Mech. 51 (1972) 357-362.

[26] M. S. Howe, The influence of vortex shedding on the generation of sound by convected turbulence, J. Fluid Mech. 76 (1976) 711-740.

[27] M. S. Howe, Theory of vortex sound, Cambridge University Press, 2002.

[28] S. A. L. Glegg, W. Devenport, Unsteady loading on an airfoil of arbitrary thickness, J. Sound Vib. 319 (2009) 1252-1270.

[29] S. M. Grace, Unsteady blade response: The BVI model vs. the gust model, in: 7th AIAA/CEAS Aeroacoustics Conference. AIAA Paper 2001-2209, 2001.

[30] H. Abou-Hussein, A. DeBenedicts, N. Harrison, M. Kim, F. Rodrigues M. A. Zagadou, M. S. Howe, Vortex-surface interaction noise: A compendium of worked examples, J. Sound Vib. 252 (2002) 883-918.

[31] C. E. Brown, W. H. Michael, Effect of leading-edge separation on the lift of a delta wing, J. Aero. Sci. 21 (1954) 690-706.

[32] M. S. Howe, Emendation of the Brown \& Michael equation with application to sound generation by vortex motion near a half-plane, J. Fluid Mech. 329 (1996) 89-101.

[33] Y. P. Guo, Application of the Ffowcs Williams/Hawkings equation to two-dimensional problems, J. Fluid Mech. 403 (2000) 201-221.

[34] A. Manela, Nonlinear effects of flow unsteadiness on the acoustic radiation of a heaving airfoil, J. Sound Vib. 332 (2013) 7076-7088. 
[35] J. Straus, P. Renzoni, R. E. Mayle, Airfoil pressure measurements during a blade vortex interaction and a comparison with theory, AIAA J. 28 (1990) 222-228.

[36] W. R. Sears, Some aspects of non-stationary airfoil theory and its practical application, J. Aeronaut. Sci. 8 (1941) 104-108.

[37] M. B. Horner, E. Saliveros, R. A. McD. Galbraith, An examination of vortex convection effects during blade-vortex interaction, Aeronaut. J. 96 (1992) 373-379.

[38] C. Kitaplioglu, F. X. Caradonna, C. L. Burley, Parallel blade-vortex interactions: an experimental study and comparison with computations, J. Am. Helicopter Soc. 42 (1997) 272-281.

[39] J. O. Bridgeman, K. Ramachandran, F. X. Caradonna, D. Prichard, A computational analysis of parallel blade-vortex interactions using vorticity embedding, in: American Helicopter Society 50th Annual Forum, Washington D.C., 1994.

[40] A. Thom, K. Duraisamy, High-resolution simulations of parallel blade-vortex interactions, AIAA J. 48 (2010) 2313-2324.

[41] F. M. White, Viscous Fluid Flow, McGraw-Hill, 1991.

[42] J. W. Kim, P. J. Morris, Computation of subsonic inviscid flow past a cone using high-order schemes, AIAA J. 40 (2002) 1961-1968.

[43] J. W. Kim, A. S. H. Lau, N. D. Sandham, CAA boundary conditions for airfoil noise due to high-frequency gusts, Proc. Eng. 6 (2010) 244-253.

[44] J. W. Kim, A. S. H. Lau, N. D. Sandham, Proposed boundary conditions for gustairfoil interaction noise, AIAA J. 48 (2010) 2705-2709.

[45] J. W. Kim, Optimised boundary compact finite difference schemes for computational aeroacoustics, J. Comput. Phys. 225 (2007) 995-1019.

[46] J. W. Kim, High-order compact filters with variable cut-off wavenumber and stable boundary treatment, Comput. Fluids 39 (2010) 1168-1182.

[47] J. W. Kim, D. J. Lee, Generalized characteristic boundary conditions for computational aeroacoustics, AIAA J. 38 (2000) 2040-2049.

[48] J. W. Kim, D. J. Lee, Generalized characteristic boundary conditions for computational aeroacoustics, part 2, AIAA J. 42 (2004) 47-55.

[49] J. W. Kim, Quasi-disjoint pentadiagonal matrix systems for the parallelization of compact finite-difference schemes and filters, J. Comput. Phys. 241 (2013) 168-194.

[50] H. C. Yee, N. D. Sandham, M. J. Djomehri, Low-dissipative high-order shockcapturing methods using characteristic-based filters, J. Comput. Phys. 150 (1999) $199-238$.

[51] M. E. Goldstein, Aeroacoustics, McGraw-Hill, 1976.

[52] J. E. Ffowcs Williams, L. Hall, Aerodynamic sound generation by turbulent flow in the vicinity of a scattering half plane, J. Fluid Mech. 40 (1970) 657-670. 
[53] R. K. Amiet, Noise due to turbulent flow past a trailing edge, J. Sound Vib. 47 (1976) 387-393.

[54] V. P. Blandeau, P. F. Joseph, G. Jenkins, C. J. Powles, Comparison of sound power radiation from isolated airfoils and cascades in turbulent flow, J. Acoust. Soc. Am. 129 (2011) 3521-3530.

[55] M. Roger, S. Moreau, Back-scattering correction and further extensions of Amiet's trailing-edge noise model. Part 1: theory, J. Sound Vib. 286 (2005) 477-506.

[56] J. E. Ffowcs Williams, D. L. Hawkings, Sound generation by turbulence and surface in arbitrary motion, Philos. Trans. R. Soc. Lond. A 264 (1969) 321-342.

[57] R. D. Sandberg, N. D. Sandham, A modification of Amiet's classical trailing edge noise theory for strictly two dimensional flows, University of Southampton: Report No. AFM-07/04 (2007).

[58] H. Schlichting, Boundary layer theory, 7th ed., McGraw-Hill, 1979.

[59] F. Farassat, Derivations of formulations 1 and 1a of Farassat, NASA/TM-2007-214853 (2007).

[60] P. Huerre, P. A. Monkewitz, Local and global instabilities in spatially developing flows, Annu. Rev. Fluid Mech. 22 (1990) 473-537.

[61] R. Jordinson, The flat flate boundary layer, part 1, numerical integration of the Orr-Sommerfeld equation, J. Fluid Mech. 43 (1970) 801-811. 